\title{
RESEARCH ARTICLE \\ Monte Carlo sampling processes and incentive compatible allocations in large economies
}

\author{
Peter J. Hammond ${ }^{1}$ (D) Lei Qiao ${ }^{2} \cdot$ Yeneng Sun $^{3}$ \\ Received: 6 November 2019 / Accepted: 6 October 2020 / Published online: 23 October 2020 \\ (c) The Author(s) 2020
}

\begin{abstract}
Monte Carlo simulation is used in Hammond and Sun (Econ Theory 36:303-325, 2008. https://doi.org/10.1007/s00199-007-0279-7) to characterize a standard stochastic framework involving a continuum of random variables that are conditionally independent given macro shocks. This paper presents some general properties of such Monte Carlo sampling processes, including their one-way Fubini extension and regular conditional independence. In addition to the almost sure convergence of Monte Carlo simulation considered in Hammond and Sun (2008), here we also consider norm convergence when the random variables are square integrable. This leads to a necessary and sufficient condition for the classical law of large numbers to hold in a general Hilbert space. Applying this analysis to large economies with asymmetric information shows that the conflict between incentive compatibility and Pareto efficiency is resolved asymptotically for almost all sampling economies, following some similar results in McLean and Postlewaite (Econometrica 70:2421-2453, 2002) and Sun and Yannelis (J Econ Theory 134:175-194, 2007. https://doi.org/10.1016/j.jet.2006.03. 001).
\end{abstract}

Keywords Law of large numbers · Monte Carlo sampling process · One-way Fubini property $\cdot$ Hilbert space $\cdot$ Incentive compatibility $\cdot$ Asymmetric information · Pareto efficiency

$凶 \quad$ Peter J. Hammond

p.j.hammond@warwick.ac.uk

Lei Qiao

qiao.lei@mail.shufe.edu.cn

Yeneng Sun

ynsun@nus.edu.sg

1 Department of Economics, University of Warwick, Coventry CV4 7AL, UK

2 School of Economics, Shanghai University of Finance and Economics, 777 Guoding Road, Shanghai 200433, China

3 Departments of Economics and Mathematics, National University of Singapore, Singapore 119076, Singapore 
JEL Classification C65 · D51 · D61 · D82

\section{Introduction}

Following the early writings by Lucas and Prescott (1974) and Bewley (1986), macroeconomists have made widespread use of a model of an economy with many agents who face individual random shocks. These shocks are typically modelled as a continuum of random variables that are conditionally independent given common macro level shocks. Proposition 4 in Hammond and Sun (2008), however, shows that in this framework, the joint measurability condition that is usually imposed on a stochastic process can be satisfied only if there is essentially no idiosyncratic risk at all. The approach of Monte Carlo simulation, initiated in Hammond and Sun (2003) for the symmetric case and extended in Hammond and Sun (2008) for the general case, can be used to characterize when, even in the absence of the usual joint measurability assumption, the standard stochastic framework for many heterogeneous agents facing individual uncertainty may still be valid. This paper provides a systematic study of the underlying Monte Carlo sampling processes. We also present an application involving allocations in large exchange economies with many asymmetrically informed consumers. In particular, we show how Monte Carlo sampling helps resolve the conflict between incentive compatibility and Pareto efficiency, which vanishes in the limit as the number of agents tends to infinity.

Let

$$
I \times \Omega \ni(i, \omega) \mapsto g_{i}(\omega) \in X
$$

be a process with a continuum of random variables, indexed by members $i$ of an atomless probability space $(I, \mathcal{I}, \lambda)$, all defined on the same sample probability space $(\Omega, \mathcal{F}, P)$, and taking values in a Polish space $X$. Let $I^{\infty}$ and $X^{\infty}$ denote the Cartesian product of infinitely many copies of the sets $I$ and $X$ respectively, with typical members $i^{\infty}=\left\langle i_{k}\right\rangle_{k=1}^{\infty}$ and $x^{\infty}=\left\langle x_{k}\right\rangle_{k=1}^{\infty}$. Then the Monte Carlo sampling process $G$ based on $g$ is a mapping

$$
I^{\infty} \times \Omega \ni\left(i^{\infty}, \omega\right) \mapsto G\left(i^{\infty}, \omega\right)=\left\langle g\left(i_{k}, \omega\right)\right\rangle_{k=1}^{\infty} \in X^{\infty}
$$

When the process $g$ has a stochastic macro structure, as defined in Sect. 2.3, Theorem 1 shows that so does the Monte Carlo sampling process $G$. In this case, the process $G$ also has the property of admitting a "one-way Fubini extension" that makes $G$ jointly measurable with respect to an extension of the usual product $\sigma$-algebra.

The Monte Carlo simulation approach in Hammond and Sun (2008) uses the almost sure convergence of the sample averages. For a square-integrable process, we also consider here the case of norm convergence. Based on the iterative extension of an infinite product measure introduced in Hammond and Sun (2006b), we formulate a "sharp" law of large numbers, requiring norm convergence of sample averages only for all sequences outside an iteratively null set, rather than a smaller classical null set. We prove that a process with square-integrable random variables satisfies this sharp law if 
and only if it is both Gel'fand-integrable and norm integrably bounded in the Hilbert space of square integrable random variables. In other words, this result characterizes those processes with square-integrable random variables whose average conditional expectation, given the macro states, can be estimated using Monte Carlo simulation.

For allocations in a finite-agent asymmetric information economy, it is well known that there is a conflict between incentive compatibility and Pareto efficiency (see, for example, Example 0.1 on p. vi of Glycopantis and Yannelis (2005)). The papers McLean and Postlewaite (2002) and Sun and Yannelis (2007) show the (approximate) consistency of incentive compatibility and efficiency by working with, respectively: (i) a large but finite set of agents; (ii) a continuum of agents. ${ }^{1}$ In this paper, given a sequence of economies that result from Monte Carlo sampling, we show that the conflict between incentive compatibility and Pareto efficiency is resolved asymptotically for almost all infinite sequences of economies. This corresponds to the asymptotic result for replica economies in McLean and Postlewaite (2002), and the exact result in Sun and Yannelis (2007) when, as in Sun (2006), private signals are generated by a process that is jointly measurable in a two-way Fubini extension.

The rest of the paper is organized as follows. Section 2 includes some basic definitions. Some general properties of the Monte Carlo sampling processes are presented in Sect. 3. Then Sect. 4 provides a necessary and sufficient condition for the classical law of large numbers to hold in a general Hilbert space. As an illustrative application, Sect. 5 shows that in a Monte Carlo sampled sequence of economies with asymmetric information, incentive compatibility and Pareto efficiency are asymptotically consistent. Additional definitions and all proofs appear in the Appendix, which is Sect. 6.

\section{Basic formulation}

\subsection{Monte Carlo sampling processes}

We model a continuum $\left\langle g_{i}\right\rangle_{i \in I}$ of random variables indexed by $i \in I$ as a process $g: I \times \Omega \rightarrow X$ where:

1. $(I, \mathcal{I}, \lambda)$ is an atomless probability space, often the Lebesgue unit interval, whose typical member is an index $i$ that identifies one particular economic agent;

2. $(\Omega, \mathcal{F}, P)$ is a probability space that represents the overall risk in the process $;^{2}$

3. $(X, \mathcal{B})$ is a Polish space with its Borel $\sigma$-algebra;

4. each indexed function $g_{i}: \Omega \rightarrow X$ is measurable, so a random variable;

A (Monte Carlo) sample of the indices $i \in I$ is a countable collection $i^{\infty}=\left\langle i_{k}\right\rangle_{k=1}^{\infty}$ drawn from the iteratively completed infinite product probability space $\left(I^{\infty}, \overline{\mathcal{I}}^{\infty}, \bar{\lambda}^{\infty}\right)$ defined in Sect. 6.1. This space was introduced in Hammond and Sun (2006b) as the

\footnotetext{
1 The compatibility of strategyproofness and Pareto efficiency had been investigated in Hammond (1979), though only in a framework like that in Aumann $(1964,1966)$ and Hildenbrand (1974) where, given the continuum of agents, the distribution of their characteristics can be described by a deterministic probabilistic measure.

2 We follow the convention that a probability space is assumed to be countably additive, as well as complete in the sense that the $\sigma$-algebra $\mathcal{F}$ includes all subsets of every $P$-null set.
} 
usual infinite product probability space $\left(I^{\infty}, \mathcal{I}^{\infty}, \lambda^{\infty}\right)$ extended so that the $\sigma$-algebra $\overline{\mathcal{I}}^{\infty}$ includes iteratively null sets.

Corresponding to each (Monte Carlo) sample $i^{\infty}=\left\langle i_{k}\right\rangle_{k=1}^{\infty}$ of indices is a countable sequence $\left\langle g_{i_{k}}\right\rangle_{k=1}^{\infty}$ of random variables. This constitutes a (Monte Carlo) sample from the continuum of random variables $\Omega \ni \omega \mapsto g_{i}(\omega) \in X$ as $i$ varies over $I$. This sample, with $i^{\infty} \in I^{\infty}$ fixed, can be regarded as part of one meta (or Monte Carlo) sampling process $G$ defined by (1).

\subsection{One-way Fubini property}

The following definition was introduced in Hammond and Sun (2006a).

Definition 1 A probability space $(I \times \Omega, \mathcal{W}, Q)$ extends the usual product probability space $(I \times \Omega, \mathcal{I} \otimes \mathcal{F}, \lambda \otimes P)$ provided that $\mathcal{W} \supseteq \mathcal{I} \otimes \mathcal{F}$, with $Q(E)=(\lambda \otimes P)(E)$ for all $E \in \mathcal{I} \otimes \mathcal{F}$.

The extended space $(I \times \Omega, \mathcal{W}, Q)$ is a one-way Fubini extension of the product probability space $(I \times \Omega, \mathcal{I} \otimes \mathcal{F}, \lambda \otimes P)$ provided that, given any $Q$-integrable function $I \times \Omega \ni(i, \omega) \mapsto f(i, \omega) \in \mathbb{R}:$

(i) for $\lambda$-almost all $i \in I$, the random variable $\omega \mapsto f_{i}(\omega)$ is integrable on $(\Omega, \mathcal{F}, P)$;

(ii) the function $i \mapsto \int_{\Omega} f_{i} d P$ is integrable on $(I, \mathcal{I}, \lambda)$, with $\int_{I \times \Omega} f d Q=$ $\int_{I}\left(\int_{\Omega} f_{i} d P\right) d \lambda$.

A process $g: I \times \Omega \rightarrow X$ is said to satisfy the one-way Fubini property if there is a one-way Fubini extension $(I \times \Omega, \mathcal{W}, Q)$ such that $g$ is $\mathcal{W}$-measurable.

\subsection{Regular conditional independence}

A $\sigma$-algebra $\mathcal{C}$ on $\Omega$ is said to be countably generated if there exists a countable family $\left\{C_{n}\right\}_{n=1}^{\infty}$ of subsets of $\Omega$ that generates $\mathcal{C}$. Given a complete probability space $(\Omega, \mathcal{F}, P)$, a sub- $\sigma$-algebra $\mathcal{C}$ of $\mathcal{F}$ is said to be countably generated if it is the strong completion of a countably generated $\sigma$-algebra $\mathcal{C}^{\prime}$, in the sense that

$$
\mathcal{C}=\left\{A \in \mathcal{F} \mid \exists A^{\prime} \in \mathcal{C}^{\prime}: P\left(A \triangle A^{\prime}\right)=0\right\}
$$

Definition 2 Let $g$ be a process from $I \times \Omega$ to the Polish space $X$ with its Borel $\sigma$-algebra $\mathcal{B}$. Let $\mathcal{C}$ be a countably generated sub- $\sigma$-algebra of $\mathcal{F}$ in the complete probability space $(\Omega, \mathcal{F}, P)$. Let $\mathcal{M}(X)$ denote the space of probability measures on the space $(X, \mathcal{B})$.

1. Two random variables $\phi$ and $\psi$ that map $(\Omega, \mathcal{F}, P)$ to $X$ are said to be conditionally independent given $\mathcal{C}$ if, for any Borel sets $B_{1}, B_{2} \in \mathcal{B}$, the conditional probabilities satisfy

$$
P\left(\phi^{-1}\left(B_{1}\right) \cap \psi^{-1}\left(B_{2}\right) \mid \mathcal{C}\right)=P\left(\phi^{-1}\left(B_{1}\right) \mid \mathcal{C}\right) P\left(\psi^{-1}\left(B_{2}\right) \mid \mathcal{C}\right)
$$

2. The process $g$ is said to be essentially pairwise conditionally independent given $\mathcal{C}$ if, for $\lambda$-a.e. $i_{1} \in I$, the random variables $g_{i_{1}}$ and $g_{i_{2}}$ are conditionally independent given $\mathcal{C}$ for $\lambda$-a.e. $i_{2} \in I$. 
3. An $\mathcal{I} \otimes \mathcal{C}$-measurable mapping $\mu$ from $I \times \Omega$ to $\mathcal{M}(X)$ is said to be an essentially regular conditional distribution process of $g$ given $\mathcal{C}$ if, for $\lambda$-a.e. $i \in I$, the $\mathcal{C}$ measurable mapping $\omega \mapsto \mu_{i \omega}$ is a regular conditional distribution $P\left(g_{i}^{-1} \mid \mathcal{C}\right)$ of the random variable $g_{i}$.

4. The process $g$ is said to be regularly conditionally independent given $\mathcal{C}$ if $g$ is essentially pairwise conditionally independent given $\mathcal{C}$, and also $g$ admits an essentially regular conditional distribution process $\mu$ given $\mathcal{C}$. In this case, we also say that $g$ admits a stochastic macro structure $(\mathcal{C}, \mu)$.

\section{Properties of Monte Carlo sampling processes}

Let $g$ be a process from $I \times \Omega$ to $X$ and $\mathcal{C}$ a countably generated sub- $\sigma$-algebra of $\mathcal{F}$ in $(\Omega, \mathcal{F}, P)$. Suppose that $g$ admits a stochastic macro structure $(\mathcal{C}, \mu)$. By Theorem 1 in Hammond and Sun (2016) (stated as Lemma 1 in Sect. 6.1 below), there exists a one-way Fubini extension $(I \times \Omega, \mathcal{W}, Q)$ such that the process $g$ is $\mathcal{W}$-measurable.

The following theorem shows that the Monte Carlo sampling process $G$ defined by (1) has the one-way Fubini property. It also shows that $G$ satisfies regular conditional independence given $\mathcal{C}$, and identifies its regular conditional distribution process.

Let $\mathcal{M}\left(X^{\infty}\right)$ denote the set of probability measures on the infinite product measurable space $\left(X^{\infty}, \mathcal{B}^{\infty}\right)$.

Theorem 1 Let $G: I^{\infty} \times \Omega \rightarrow X^{\infty}$ be a Monte Carlo sampling process based on $g$. Then there exists a one-way Fubini extension $\left(I^{\infty} \times \Omega, \tilde{\mathcal{W}}, \tilde{Q}\right)$ of $\left(I^{\infty} \times \Omega, \overline{\mathcal{I}}^{\infty} \otimes\right.$ $\mathcal{F}, \bar{\lambda}^{\infty} \otimes P$ ) such that $G$ is $\tilde{\mathcal{W}}$-measurable. In addition, $G$ is essentially pairwise conditionally independent given $\mathcal{C}$. It also admits the essentially regular conditional distribution process $\bar{\mu}$ defined by

$$
I^{\infty} \times \Omega \ni\left(i^{\infty} \omega\right) \mapsto \bar{\mu}_{i \infty \omega}:=\otimes_{k=1}^{\infty} \mu_{i_{k} \omega} \in \mathcal{M}\left(X^{\infty}\right)
$$

\section{Characterizing a sharp law of large numbers}

Let $g$ be a process from $I \times \Omega$ to $X$ as in Sect. 3, and let $h: I \times X \rightarrow \mathbb{R}$ be an $\mathcal{I} \otimes \mathcal{B}$-measurable function with $\int_{I}\left[\int_{\Omega} h_{i}^{2}\left(g_{i}(\omega)\right) d P\right] d \lambda<\infty$. Then Lemma 11 in Hammond and Sun (2008) says that for $\bar{\lambda}^{\infty}$-a.e. $i^{\infty} \in I^{\infty}$, one has

$$
\frac{1}{n} \sum_{k=1}^{n} h\left(i_{k}, g\left(i_{k}, \omega\right)\right) \rightarrow \int_{I}\left[\int_{X} h(i, x) d \mu_{i \omega}\right] d \lambda \text { for } P \text {-almost all } \omega \in \Omega
$$

Under the framework of one-way Fubini extension, we have the following corollary.

Corollary 1 Let $G: I^{\infty} \times \Omega \rightarrow X^{\infty}$ be the Monte Carlo sampling process based on g. Suppose that $\left(I^{\infty} \times \Omega, \tilde{\mathcal{W}}, \tilde{Q}\right)$ is a one-way Fubini extension of $\left(I^{\infty} \times \Omega, \overline{\mathcal{I}}^{\infty} \otimes\right.$ $\left.\mathcal{F}, \bar{\lambda}^{\infty} \otimes P\right)$ such that $G$ is $\tilde{\mathcal{W}}$-measurable. Then for $\tilde{Q}$-almost all $\left(i^{\infty}, \omega\right) \in I^{\infty} \times \Omega$, 
one has

$$
\frac{1}{n} \sum_{k=1}^{n} h\left(i_{k}, g\left(i_{k}, \omega\right)\right) \rightarrow \int_{I}\left[\int_{X} h(i, x) d \mu_{i \omega}\right] d \lambda
$$

Let $L^{2}(P)$ be the space of real-valued square integrable functions on $(\Omega, \mathcal{F}, P)$, made into a Hilbert space by defining, for any pair $\varphi, \psi \in L^{2}(P)$, the standard inner product $\langle\varphi, \psi\rangle:=\int_{\Omega} \varphi(\omega) \psi(\omega) d P$. For each fixed $i \in I$, define the random variable $f(i)(\cdot)$ so that

$$
\Omega \ni \omega \mapsto f(i)(\omega)=h(i, g(i, \omega)) \in \mathbb{R}
$$

The assumption that $\int_{I}\left[\int_{\Omega} h_{i}^{2}\left(g_{i}(\omega)\right) d P\right] d \lambda<\infty$ implies that for $\lambda$-almost all $i \in I$, the random variable $f(i)$ is an element in the Hilbert space $L^{2}(P)$. Corollary 1 indicates that the sample average $\frac{1}{n} \sum_{k=1}^{n} f\left(i_{k}\right)(\omega)$ converges $\tilde{Q}$-almost surely to $\int_{I}\left[\int_{X} h(i, x) d \mu_{i \omega}\right] d \lambda$. Since the function $I \ni i \mapsto f(i)$ takes values in the Hilbert space $L^{2}(P)$, a natural question is whether one can obtain a similar result for convergence in the norm of $L^{2}(P)$. This is answered in the following proposition.

Proposition 1 For $\bar{\lambda}^{\infty}$-almost all $i^{\infty} \in I^{\infty}$, one has

$$
\left\|\frac{1}{n} \sum_{k=1}^{n} f\left(i_{k}\right)-\int_{I}\left[\int_{X} h(i, x) d \mu_{i \omega}\right] d \lambda\right\| \rightarrow 0
$$

where the random variable $\omega \mapsto \int_{I}\left[\int_{X} h(i, x) d \mu_{i \omega}\right] d \lambda$ is in $L^{2}(P)$, and $\|\cdot\|$ denotes the standard norm on the Hilbert space $L^{2}(P)$.

This result can be viewed as the classical law of large numbers for a sequence of random variables taking values in the Hilbert space $L^{2}(P)$. One may wonder whether such a result can be extended to other Hilbert spaces, or to Banach spaces more generally.

Let $\mathbb{B}$ be a Banach space, with norm $\|\cdot\|$ and norm dual $\mathbb{B}^{\prime}$. Given any $b \in \mathbb{B}$ and $b^{\prime} \in \mathbb{B}^{\prime}$, let $\left\langle b, b^{\prime}\right\rangle$ denote the real value of the continuous linear mapping $b^{\prime}$ evaluated at $b$. In the case when $\mathbb{B}$ is a Hilbert space, we shall denote it by $\mathbb{H}$. Then, of course, $\left\langle b, b^{\prime}\right\rangle$ can be regarded as the inner product.

Henceforth we use the respective abbreviations LLN and SLLN for the law of large numbers, and the sharp law of large numbers.

Definition 3 Let $f$ be a function from $(I, \mathcal{I}, \lambda)$ to a Banach space $\mathbb{B}$.

1. The function $f$ is said to satisfy LLN (resp., SLLN) if there exists $a \in \mathbb{B}$ such that $\left\|a-\frac{1}{n} \sum_{k=1}^{n} f\left(i_{k}\right)\right\| \rightarrow 0 \lambda^{\infty}$-a.s. (resp., $\bar{\lambda}^{\infty}$-a.s.). Let LLN(BB) (resp., SLLN(BB) denote the (linear) space of all functions from $I$ to $\mathbb{B}$ that satisfy LLN (resp., SLLN).

2. The function $f$ is said to be Gel'fand integrable if there exists a vector $b \in \mathbb{B}$ called the Gel'fand integral of $f$ such that, for all $b^{\prime} \in \mathbb{B}^{\prime}$, the real-valued function $i \mapsto\left\langle f(\cdot), b^{\prime}\right\rangle$ on $I$ is $\lambda$-integrable, with $\int_{I}\left\langle f(i), b^{\prime}\right\rangle d \lambda=\left\langle b, b^{\prime}\right\rangle .^{3}$

\footnotetext{
3 This follows the terminology of Dobric (1987) and Hoffmann-Jørgensen (1985). When the Gel'fand integral of $1_{S} f$ is defined for every $S \in \mathcal{I}$, this is often called the Pettis integral - see, for example,
} 
3. A function $f^{*}: I \rightarrow \mathbb{R}_{+}$norm dominates $f: I \rightarrow \mathbb{B}$ if $\|f(i)\| \leq f^{*}(i)$ for $\lambda$-a.e. $i \in I$. The function $f$ is said to be norm integrably bounded if there exists a $\lambda$-integrable function $f^{*}: I \rightarrow \mathbb{R}_{+}$that norm dominates $f$.

From now on, let $\mathcal{L}(\lambda, \mathbb{B})$ denote the (linear) space of all functions $f$ from $(I, \mathcal{I}, \lambda)$ to $\mathbb{B}$ that are both Gel'fand integrable and norm integrably bounded. The following Proposition is well known in the literature on random variables with values in a Banach space. For Part (1), see for example Hoffmann-Jørgensen (1985, Theorem 2.4). Part (2) is taken from Dobric (1987, Theorem 3.1).

Proposition 21 . $\operatorname{LLN}(\mathbb{B}) \subseteq \mathcal{L}(\lambda, \mathbb{B})$ for any Banach space $\mathbb{B}$.

2. Suppose that $I$ is a Polish space, that $\mathcal{I}$ is its Borel $\sigma$-algebra, and that $\lambda$ is an atomless probability measure. There is a Hilbert space $\mathbb{H}$ such that $\mathcal{L}(\lambda, \mathbb{H})$ is not equal to $\operatorname{LLN}(\mathbb{H})$.

Part (1) of Proposition 2 says that a necessary condition for $f$ to satisfy the usual LLN is that $f$ must be both Gel'fand integrable and norm integrably bounded. On the other hand, Part (2) of Proposition 2 shows that these two conditions are not sufficient for the LLN to hold, even for the special case of a Hilbert space. It means that LLN $(\mathbb{B})$ is in general a proper subset of $\mathcal{L}(\lambda, \mathbb{B})$.

Since a $\lambda^{\infty}$-null set is automatically $\bar{\lambda}^{\infty}$-null, any function in $\operatorname{LLN}(\mathbb{B})$ must be in $\operatorname{SLLN}(\mathbb{B})$. Given that the set $\operatorname{SLLN}(\mathbb{B})$ is bigger than the set $\operatorname{LLN}(\mathbb{B})$, two natural questions arise. The first is whether the inclusion relationship in Part (1) of Proposition 2 still holds. The second is when such an inclusion can be strengthened to an equality.

The following theorem shows that when the product measure $\lambda^{\infty}$ is extended to its iterated completion $\bar{\lambda}^{\infty}$, not only does the strengthened inclusion $\operatorname{SLLN}(\mathbb{B}) \subseteq \mathcal{L}(\lambda, \mathbb{B})$ hold for a general Banach space $\mathbb{B}$, but it becomes an equality in the Hilbert space case. This equality provides a very general characterization of the functions from $(I, \mathcal{I}, \lambda)$ to a general Hilbert space $\mathbb{H}$ that satisfy our sharp law of large numbers in the iterated completion of the product probability space. Moreover, an obvious corollary of our results is that $\operatorname{LLN}(\mathbb{B})$ is in general a proper subset of $\operatorname{SLLN}(\mathbb{B})$, even when $\mathbb{B}$ is a Hilbert space.

Theorem 2 (Sharp law of large numbers) If $f$ is any function mapping $(I, \mathcal{I}, \lambda)$ to a Banach space $\mathbb{B}$ for which $S L L N$ is satisfied, then $f \in \mathcal{L}(\lambda, \mathbb{B})$; that is, $\operatorname{SLLN}(\mathbb{B}) \subseteq$ $\mathcal{L}(\lambda, \mathbb{B})$. More importantly, if $\mathbb{B}$ is a Hilbert space $\mathbb{H}$, then $f$ satisfies SLLN if and only if $f$ is Gel'fand integrable and norm integrably bounded. That is, $\operatorname{SLLN}(\mathbb{H})=\mathcal{L}(\lambda, \mathbb{H})$.

Consider the function $f$ from $(I, \mathcal{I}, \lambda)$ to the Hilbert space $L^{2}(P)$ which is defined by (4). The function $f$ is obviously norm integrably bounded because $\|f(i)\|=$ $\left(\int_{\Omega} h_{i}^{2}\left(g_{i}(\omega)\right) d P\right)^{\frac{1}{2}}$ and $\int_{I}\left[\int_{\Omega} h_{i}^{2}\left(g_{i}(\omega)\right) d P\right] d \lambda<\infty$. The following claim, which says that such a function $f$ is also Gel'fand integrable, indicates why Proposition 1 is a special case of Theorem 2 .

Claim 1 Let $f$ be the function from $(I, \mathcal{I}, \lambda)$ to $L^{2}(P)$ defined as in equation (4) by $f(i)(\omega)=h(i, g(i, \omega))$. Then $f$ is Gel'fand integrable.

Aliprantis and Border (1999) and Diestel and Uhl (1977, p. 53). Note that $1_{S}$ is the indicator function of the set $S$. 


\section{Allocations in large economies with asymmetric information}

\subsection{The information structure}

We use the same information structure as that set out in Sun and Yannelis (2007, $2008)$. Suppose that the fixed atomless probability space $(I, \mathcal{I}, \lambda)$ represents the space of economic agents. Let $S=\left\{s_{1}, s_{2}, \ldots, s_{K}\right\}$ denote the finite set of true states of nature (with power set denoted by $\mathcal{S}$ ). We assume that these are not known by any agent. Let $T^{0}=\left\{q_{1}, q_{2}, \ldots, q_{L}\right\}$ denote the space of all possible signals (or types) for each individual agent. We consider the measurable space $(T, \mathcal{T})$ of private signal or type profiles for all the agents $i \in I$. Thus, $T$ is a subset of $\left(T^{0}\right)^{I}$, the space of all functions from $I$ to $T^{0}{ }^{4}$ For each agent $i \in I$, the function value $t(i)$ (also denoted by $t_{i}$ ) is agent $i$ 's private signal, whereas $t_{-i}$ is the restriction of the signal profile $t$ to the set $I \backslash\{i\}$ of agents different from $i$; let $T_{-i}$ denote the set of all such $t_{-i}$. For simplicity, we assume that $(T, \mathcal{T})$ has a rich enough product structure so that $T$ is a product of $T_{-i}$ and $T^{0}$, whereas $\mathcal{T}$ is the product $\sigma$-algebra of the power set $\mathcal{T}^{0}$ on $T^{0}$ with a $\sigma$-algebra $\mathcal{T}_{-i}$ on $T_{-i}$. Given any $t \in T$ and $t_{i}^{\prime} \in T^{0}$, we shall adopt the usual notation $\left(t_{-i}, t_{i}^{\prime}\right)$ to denote the signal profile whose value is $t_{i}^{\prime}$ for agent $i$, but the same as $t_{j}$ for all other agents $j \in I \backslash\{i\}$.

To represent all the uncertainty about the true states as well as the agents' signals, we consider the probability space $(\Omega, \mathcal{F}, P)$ where $(\Omega, \mathcal{F})$ is the product measurable space $(S \times T, \mathcal{S} \otimes \mathcal{T})$. Let $P^{S}$ and $P^{T}$ be the marginal probability measures of $P$ on $(S, \mathcal{S})$ and $(T, \mathcal{T})$ respectively. For each $i \in I$, let $\tilde{s}$ and $\tilde{t}_{i}$ denote the projection mappings from $\Omega$ to $S$ and to $T^{0}$ respectively, with $\tilde{t}_{i}(s, t)=t_{i}{ }^{5}$ After excluding any $P^{S}$-null state, we assume without loss of generality that each true state $s \in S$ is non-null in the sense that $\pi_{s}:=P^{S}(\{s\})>0$; let $P_{s}^{T}$ be the conditional probability measure on $(T, \mathcal{T})$ given that the random variable $\tilde{s}$ takes value $s$. Thus, for each $B \in \mathcal{T}$, one has $P_{s}^{T}(B)=P(\{s\} \times B) / \pi_{s}$. It is obvious that $P^{T}=\sum_{s \in S} \pi_{s} P_{s}^{T}$. Note that in the literature the conditional probability measure $P_{S}^{T}$ is often denoted as $P(\cdot \mid s)$.

For each fixed $t \in T$, define also the conditional probability measure $P^{S}(\cdot \mid t)$ on $S$ so that for each fixed $s \in S$, the mapping $T \ni t \mapsto P^{S}(\{s\} \mid t)$ is $\mathcal{T}$-measurable, with $P(\{s\} \times B)=\int_{B} P^{S}(\{s\} \mid t) d P^{T}$ for each $B \in \mathcal{T}$. Let $T \ni t \mapsto p_{s}(t) \in \mathbb{R}_{+}$be the density function of $P_{s}^{T}$ with respect to $P^{T}$; it is easy to see that $P^{S}(\{s\} \mid t)=\pi_{s} p_{s}(t)$ for $P^{T}$-almost all $t \in T$.

For each $i \in I$, let $\tau_{i}$ denote the marginal signal distribution of agent $i$ on the space $T^{0}$; it is defined so that for all $q \in T^{0}$, the probability $P\left(\tilde{t}_{i}=q\right)$ equals $\tau_{i}(\{q\})$. Let $P^{S \times T_{-i}}\left(\cdot \mid t_{i}\right)$ denote the conditional probability measure on the product measurable space $\left(S \times T_{-i}, \mathcal{S} \otimes \mathcal{T}_{-i}\right)$ given that agent $i$ 's signal is $t_{i} \in T^{0}$. For any $t_{i} \in T^{0}$ with marginal probability $\tau_{i}\left(\left\{t_{i}\right\}\right)>0$, it is clear that for any $E \in \mathcal{S} \otimes \mathcal{T}_{-i}$, one has $P^{S \times T_{-i}}\left(E \mid t_{i}\right)=P\left(E \times\left\{t_{i}\right\}\right) / \tau_{i}\left(\left\{t_{i}\right\}\right)$. Denote by $\mathcal{C}$ the completed sub- $\sigma$-algebra

\footnotetext{
4 The standard literature usually assumes that different agents have different sets of possible signals, all of which occur with positive probability. For notational simplicity, we choose to work instead with a common set $T^{0}$ of possible signals, but allow some of these to have zero probability for some agents. There is no loss of generality in this latter approach.
}

5 Because $\Omega=S \times T$, the mapping $\tilde{t}_{i}$ can also be viewed as a projection from $T$ to $T^{0}$. 
of $\mathcal{F}=\mathcal{S} \otimes \mathcal{T}$ on $\Omega=S \times T$ that is generated by the union of the finite family $\{\{s\} \times T: s \in S\}$ with the set of all $P$-null subsets of $S \times T$.

Let $f$ denote the private signal process from $I \times \Omega$ to the finite type space $T^{0}$ defined so that $f(i, \omega)=\tilde{t}_{i}(\omega)$. Typically, for each $\omega \in \Omega$ the mapping $i \mapsto$ $f(i, \omega)$ will not be $\mathcal{I}$-measurable. Assume that $f$ is essentially pairwise conditionally independent given $\mathcal{C}$, and also admits an essentially regular conditional distribution process $\mu$ given $\mathcal{C}$. By definition of the latter, we know that for $\lambda$-almost all $i \in I$, the marginal process $\Omega \in \omega \mapsto \mu_{i}(\omega) \in \Delta\left(T^{0}\right)$ is a regular conditional distribution of $\Omega \in \omega \mapsto f_{i}(\omega) \in T^{0}$ given $\mathcal{C}$.

Let $\bar{\mu}:=\int_{I} \mu_{i} d \lambda$ be the mean conditional signal distribution over all agents. Then the Fubini property implies that $\bar{\mu}$ is a $\mathcal{C}$-measurable mapping from $\Omega$ to $\Delta\left(T^{0}\right)$. We assume that the process $f$ is non-trivial in the sense that $\mathcal{C}$ is the same as the completed sub- $\sigma$-algebra of $\mathcal{F}$ generated by $\bar{\mu}$ together with the $P$-null subsets of $\Omega$. This means that the mean conditional signal distribution carries the same information as the true state.

Let $\left\{A_{s}\right\}_{s \in S}$ be the $\mathcal{C}$-measurable partition of $\Omega$ such that $\tilde{s}(\omega)=s$ for any $\omega \in A_{s}$. Then $\mathcal{C}$ is generated by the finite family $\left\{A_{s}\right\}_{s \in S}$. Since $\bar{\mu}$ is $\mathcal{C}$-measurable, there exists a corresponding finite collection of measures $\left\{\mu_{s}\right\}_{s \in S}$ in $\Delta\left(T^{0}\right)$ such that $\bar{\mu}(\omega)=$ $\sum_{s \in S} 1_{A_{s}}(\omega) \mu_{s}$ for $P$-almost all $\omega \in \Omega$. It is clear that $\mu_{s}$ is the agents' average signal distribution conditional on the true state being $s$. The non-triviality assumption above implies that

$$
\forall s, s^{\prime} \in S, \quad s \neq s^{\prime} \Longrightarrow \mu_{s} \neq \mu_{s^{\prime}}
$$

\subsection{A state contingent large economy}

First, we define a complete information economy $\mathcal{E}^{c}$. The common consumption set of each agent $i \in I$ is the positive orthant $\mathbb{R}_{+}^{m}$. Suppose that for any given $i \in I$ and true state $s \in S$, the mapping $\mathbb{R}_{+}^{m} \ni x \mapsto u_{i}(x ; s)$ is agent $i$ 's utility function when the state is $s$. For any given $i \in I$ and $s \in S$, assume that $\mathbb{R}_{+}^{m} \ni x \mapsto u_{i}(x ; s) \in \mathbb{R}$ is continuous and strictly monotonic in $x \in \mathbb{R}_{+}^{m}$ in the sense that

$$
\tilde{x} \geqq x \text { and } \tilde{x} \neq x \Longrightarrow u_{i}(\tilde{x} ; s)>u_{i}(x ; s)
$$

Assume too that for any fixed $x \in \mathbb{R}_{+}^{m}$ and $s \in S$, the mapping $I \ni i \mapsto u_{i}(x ; s)$ is $\mathcal{I}$-measurable in $i \in I^{6}$

In this section, let $\|x\|$ denote the Euclidean norm of any vector $x \in \mathbb{R}^{m}$. Assume also that, in addition to continuity of each individual's utility function $\mathbb{R}_{+}^{m} \ni x \mapsto$ $u_{i}(x ; s)$, the entire family of utility functions $\mathbb{R}_{+}^{m} \ni x \mapsto u_{i}(x ; s)$ as $(i, s)$ varies over $I \times S$ is uniformly equicontinuous in the sense that, for any $\epsilon>0$, there exists $\delta>0$ such that $\|x-\tilde{x}\|<\delta$ implies $|u(i, x, s)-u(i, \tilde{x}, s)|<\epsilon$ for all $i \in I$, all $x, \tilde{x} \in \mathbb{R}_{+}^{m}$, and all $s \in S$.

Let $I \ni i \mapsto e(i) \in \mathbb{R}_{+}^{m}$ be the $\lambda$-integrable endowment function specifying each agent $i$ 's initial endowment. Assume that the mean endowment vector $\bar{e}:=\int_{I} e(i) d \lambda$

\footnotetext{
6 In the sequel, we shall often use subscripts to denote some argument of a function that is viewed as a parameter in a particular context.
} 
satisfies $\bar{e} \gg 0$, meaning that the mean endowment of each good is positive. Let $\Delta_{m}$ denote the unit simplex in $\mathbb{R}_{+}^{m}$.

For each $s \in S$, the collection $\mathcal{E}_{s}^{c}=\left\{(I, \mathcal{I}, \lambda), u_{s}^{I}, e^{I}\right\}$, consisting of an atomless probability space of agents with their respective utility functions $x \mapsto u_{i}(x ; s)$ and endowment vectors $e_{i}$, together constitutes a large deterministic exchange economy. A complete information economy is a collection $\mathcal{E}^{c}=\left\{\mathcal{E}_{s}^{c}: s \in S\right\}$ specifying the deterministic economy $\mathcal{E}_{s}^{c}$ for each $s \in S$. The following provides the definition of the basic concept of a Walrasian allocation.

Definition 4 1. An allocation for $\mathcal{E}^{c}$ is a function

$$
I \times S \ni(i, s) \mapsto x_{s}^{c}(i) \in \mathbb{R}_{+}^{m}
$$

such that for any fixed $s \in S$, the mapping $i \mapsto x_{s}^{c}(i)$ is $\lambda$-integrable.

2. An allocation $(i, s) \mapsto x_{s}^{c}(i)$ is feasible in $\mathcal{E}^{c}$ if, for each $s \in S$, one has $\int_{I} x_{s}^{c}(i) d \lambda=\int_{I} e(i) d \lambda$ (i.e., $x_{s}^{c}$ is feasible in $\mathcal{E}_{s}^{c}$ ).

3. A feasible allocation $(i, s) \mapsto x_{s}^{c}(i)$ is a Walrasian (or competitive equilibrium) allocation in $\mathcal{E}^{c}$ if for each $s \in S$, there is a price system $p_{s} \in \Delta_{m}$ which, together with the feasible allocation $x_{s}^{c}$, makes $\left(x_{s}^{c}, p_{s}\right)$ a competitive or Walrasian equilibrium in the large deterministic economy $\mathcal{E}_{s}^{c}$, in the sense that for $\lambda$-a.e. $i \in I$, given $i$ 's Walrasian budget set

$$
B_{i}\left(p_{s}\right):=\left\{x \in \mathbb{R}_{+}^{m}: p_{s} \cdot x \leq p_{s} \cdot e(i)\right\}
$$

one has

$$
x_{s}^{c}(i) \in \arg \max _{x}\left\{u_{i}(x ; s): x \in B_{i}\left(p_{s}\right)\right\}
$$

\subsection{Monte Carlo sampling economies}

We shall now apply Monte Carlo sampling to economies with a continuum of agents who have asymmetric information. Each agent $i \in I$ is informed about her private signal $t_{i} \in T^{0}$, but not the true state $s \in S$. Fix any $i^{\infty} \in I^{\infty}$ drawn from the iteratively completed infinite product probability space $\left(I^{\infty}, \overline{\mathcal{I}}^{\infty}, \bar{\lambda}^{\infty}\right)$. In the asymmetric information Monto Carlo sampling economy $\mathcal{E}^{i^{\infty}}$, there is a countable set of sampled agents $i^{\infty} \in I^{\infty}$.

For any $x \in \mathbb{R}_{+}^{m}$ and $t \in T$, let $U_{i}(x \mid t):=\sum_{s \in S} u_{i}(x ; s) P^{S}(\{s\} \mid t)$ denote agent $i$ 's conditional expected utility of consumption bundle $x$ given the type $t$.

A function $z$ from $(T, \mathcal{T})$ to $\mathbb{R}_{+}^{m}$ is said to be a consumption plan in $\mathcal{E}^{i \infty}$ if for any pair $t, t^{\prime} \in T$ of type profiles that coincide on $i^{\infty}$, one has $z(t)=z\left(t^{\prime}\right)$. That is, a consumption plan only depends on reported types of agents in the set $I\left(i^{\infty}\right)$ defined by

$$
I\left(i^{\infty}\right):=\cup_{k=1}^{\infty}\left\{i_{k}\right\}
$$


Let $C P\left(i^{\infty}\right)$ be the space of consumption plans in $\mathcal{E}^{i^{\infty}}$. For any agent $i \in I\left(i^{\infty}\right)$ and a consumption plan $z \in C P\left(i^{\infty}\right)$, let

$$
U_{i}(z):=\int_{\Omega} u_{i}(z(t) ; s) d P=\sum_{s \in S} \pi_{s} \int_{T} u_{i}(z(t) ; s) d P_{s}^{T}
$$

be the overall expected utility of agent $i$ for the consumption plan $t \mapsto z(t)$.

An allocation in $\mathcal{E}^{i^{\infty}}$ is a function $I^{\infty} \ni i^{\infty} \mapsto x^{i^{\infty}} \in C P\left(i^{\infty}\right)$. For any allocation $x^{i^{\infty}}$, any agent $i \in I\left(i^{\infty}\right)$, and any pair of private signals $t_{i}, t_{i}^{\prime} \in T^{0}$, let

$$
U_{i}^{i^{\infty}}\left(x_{i}^{i^{\infty}}, t_{i}^{\prime} \mid t_{i}\right):=\int_{S \times T_{-i}} u\left(i, x_{i}^{i^{\infty}}\left(t_{-i}, t_{i}^{\prime}\right), s\right) d P^{S \times T_{-i}}\left(\cdot \mid t_{i}\right)
$$

denote agent $i$ 's conditional expected utility when she receives the private signal $t_{i}$ but mis-reports it as $t_{i}^{\prime}$.

\subsection{Asymptotically feasible and constrained Pareto efficient allocations}

To discuss incentive compatibility, we invoke the revelation principle due to Dasgupta et al. (1979) and Myerson (1982), but extended in an obvious way to a continuum of consumers. That is, we consider a direct revelation mechanism in which reporting one's type truthfully is a Bayesian equilibrium for every agent in the corresponding game of incomplete information. Specifically, let $g$ denote the agents' joint reporting process $I \times T \ni(i, t) \mapsto g(i, t) \in T^{0}$ with $g(i, t)=\tilde{t}(i)$ for all $(i, t) \in I \times T$. Let $G: I^{\infty} \times T \rightarrow\left(T^{0}\right)^{\infty}$ be the corresponding Monte Carlo sampling process based on $g$. The following claim, which will be proved in Sect. 6.4, shows that $g$ also has a stochastic macro structure.

Claim 2 There is a countably generated sub-sigma-algebra $\mathcal{C}^{\prime}$ of $\mathcal{T}$ such that $g$ is regularly conditionally independent given $\mathcal{C}^{\prime}$.

By Theorem 1, this implies that the space $\left(I^{\infty} \times T, \overline{\mathcal{I}}^{\infty} \otimes \mathcal{T}, \bar{\lambda}^{\infty} \otimes P^{T}\right)$ has a one-way Fubini extension $\left(I^{\infty} \times T, \tilde{\mathcal{W}}, \tilde{Q}\right)$ such that $G$ is $\tilde{\mathcal{W}}$-measurable.

Definition 5 1. An allocation mechanism is a mapping

$$
I^{\infty} \times I \times T \ni\left(i^{\infty}, i, t\right) \mapsto x^{i^{\infty}}(i, t) \in \mathbb{R}_{+}^{m}
$$

2. The allocation mechanism $\left(i^{\infty}, i, t\right) \mapsto x^{i^{\infty}}(i, t)$ is asymptotically feasible if, for $\tilde{Q}$-almost all $\left(i^{\infty}, t\right) \in I^{\infty} \times T$, one has

$$
\left\|\frac{1}{n} \sum_{k=1}^{n} x^{i^{\infty}}\left(i_{k}, t\right)-\frac{1}{n} \sum_{k=1}^{n} e\left(i_{k}\right)\right\| \rightarrow 0 \text { as } n \rightarrow \infty
$$


3. The allocation mechanism $\left(i^{\infty}, i, t\right) \mapsto x^{i^{\infty}}(i, t)$ is incentive compatible if, for $\tilde{Q}$-almost all $\left(i^{\infty}, t\right) \in I^{\infty} \times T$, the incentive constraint $U_{i}^{i^{\infty}}\left(x_{i}^{i^{\infty}}, t \mid t\right) \geq$ $U_{i}^{i^{\infty}}\left(x_{i}^{i^{\infty}}, t^{\prime} \mid t\right)$ holds for any $i \in I\left(i^{\infty}\right)$ and any $t^{\prime} \in T^{0}$.

4. The allocation mechanism $\left(i^{\infty}, i, t\right) \mapsto x^{i^{\infty}}(i, t)$ is ex post individually rational if, for $\tilde{Q}$-almost all $\left(i^{\infty}, t\right) \in I^{\infty} \times T$, one has $U_{i}^{i^{\infty}}\left(x_{i}^{i^{\infty}} \mid t\right) \geq U_{i}^{i^{\infty}}\left(e_{i} \mid t\right)$.

5. The allocation mechanism $\left(i^{\infty}, i, t\right) \mapsto x^{i^{\infty}}(i, t)$ is asymptotically Pareto efficient if, for $\tilde{Q}$-almost all $\left(i^{\infty}, t\right) \in I^{\infty} \times T$, the following holds: for any $\epsilon>0$, there is no sequence $y: \mathbb{N} \rightarrow \mathbb{R}_{+}^{m}$ such that: (i) as $n \rightarrow \infty$, so $\left\|\frac{1}{n} \sum_{k=1}^{n} y_{k}-\frac{1}{n} \sum_{k=1}^{n} e\left(i_{k}\right)\right\| \rightarrow 0$; (ii) for any $i \in I\left(i^{\infty}\right)$, one has $U_{i}^{i^{\infty}}\left(y_{i} \mid t\right) \geq$ $U_{i}^{i^{\infty}}\left(x_{i}^{i^{\infty}} \mid t\right)+\epsilon$.

Now we are ready to state the following result for economies generated by Monto Carlo sampling.

Theorem 3 There exists an allocation mechanism $\left(i^{\infty}, i, t\right) \mapsto x^{i^{\infty}}(i, t)$ which is asymptotically feasible, incentive compatible, ex post individually rational, and asymptotically Pareto efficient.

Theorems 1 and 2 in Sun and Yannelis (2007) demonstrate the consistency of exact incentive compatibility and exact efficiency for a continuum of agents, where private signals are generated by a process that is jointly measurable in a two-way Fubini extension, as in Sun (2006). ${ }^{7}$ This paper only works in the framework of a one-way Fubini extension. Approximate versions of such a consistency result are considered in McLean and Postlewaite (2002), in Theorem 3 of Sun and Yannelis (2007), and in Theorem 3 above. Exact incentive compatibility and approximate efficiency for a special sequence of replica economies constructed from a fixed finite-agent economy with concave utilities are considered in Theorem 2 of McLean and Postlewaite (2002). Theorem 3 of Sun and Yannelis (2007) considers approximate incentive compatibility and approximate efficiency for a general sequence of large, but finite economies with possibly non-concave utilities. In the setting of a sequence of economies that result from Monte Carlo sampling, this paper shows the consistency of exact incentive compatibility and approximate efficiency for almost all infinite sequences of economies, with utilities that are allowed to be non-concave. ${ }^{8}$

Acknowledgements For their helpful comments, the authors are very grateful to the editors, notably Bernard Cornet and Nicholas Yannelis, as well as to the anonymous referees.

Open Access This article is licensed under a Creative Commons Attribution 4.0 International License, which permits use, sharing, adaptation, distribution and reproduction in any medium or format, as long as you give appropriate credit to the original author(s) and the source, provide a link to the Creative Commons licence, and indicate if changes were made. The images or other third party material in this article are included in the article's Creative Commons licence, unless indicated otherwise in a credit line to the material. If

\footnotetext{
7 Sun (2006, Proposition 5.6) shows the existence of rich Fubini extensions; see also Sun and Zhang (2009) and Podczeck (2010) for other constructions of rich Fubini extensions.

${ }^{8} \mathrm{He}$ and Yannelis (2016) consider large economies with discontinuous preferences. It remains to be seen how the results presented here as well as in Sun and Yannelis (2007) can be generalized to such a setting.
} 
material is not included in the article's Creative Commons licence and your intended use is not permitted by statutory regulation or exceeds the permitted use, you will need to obtain permission directly from the copyright holder. To view a copy of this licence, visit http://creativecommons.org/licenses/by/4.0/.

\section{Appendix}

\subsection{Some technical background}

In this subsection, we first define iteratively complete products as in Hammond and Sun (2006b). Then, for the convenience of the reader, we state as lemmas two results from Hammond and Sun (2016) that are used in this paper.

Let $\left(I_{k}, \mathcal{I}_{k}, \lambda_{k}\right)(k \in \mathbb{N})$ be a sequence of probability spaces. We use the same notation whether or not the spaces $\mathcal{P}_{k}=\left(I_{k}, \mathcal{I}_{k}, \lambda_{k}\right)$ are identical copies of a fixed space $(I, \mathcal{I}, \lambda)$. Let

$$
\mathcal{P}^{n}:=\prod_{k=1}^{n} \mathcal{P}_{k}=\left(I^{n}, \mathcal{I}^{n}, \lambda^{n}\right):=\left(\prod_{k=1}^{n} I_{k}, \bigotimes_{k=1}^{n} \mathcal{I}_{k}, \bigotimes_{k=1}^{n} \lambda_{k}\right)
$$

denote the respective $n$-fold product, and let

$$
P^{\infty}:=\prod_{k=1}^{\infty} \mathcal{P}_{k}=\left(I^{\infty}, \mathcal{I}^{\infty}, \lambda^{\infty}\right):=\left(\prod_{k=1}^{\infty} I_{k}, \bigotimes_{k=1}^{\infty} \mathcal{I}_{k}, \bigotimes_{k=1}^{\infty} \lambda_{k}\right)
$$

denote the infinite product counterpart.

The following definition is taken from Hammond and Sun (2006b).

Definition 6 A subset $E$ of the $n$-fold Cartesian product set $I^{n}$ is said to be iteratively null in $\mathcal{P}^{n}$ if for every permutation $\pi$ on $\{1, \ldots, n\}$, the $n$-fold iterated integral

$$
\int_{i_{\pi(1)} \in I_{\pi(1)}} \ldots \int_{i_{\pi(n)} \in I_{\pi(n)}} 1_{E}\left(i_{1}, i_{2}, \ldots, i_{n}\right) d \lambda_{\pi(n)}\left(i_{\pi(n)}\right) \ldots d \lambda_{\pi(1)}\left(i_{\pi(1)}\right)
$$

of the indicator function $I^{n} \ni i^{n} \mapsto 1_{E}\left(i^{n}\right) \in\{0,1\}$ for the set $E$ is well-defined and has value zero; in other words, for $\lambda_{\pi(1)}$-a.e. $i_{\pi(1)} \in I_{\pi(1)}, \lambda_{\pi(2)}$-a.e. $i_{\pi(2)} \in I_{\pi(2)}, \ldots$, $\lambda_{\pi(n)}$-a.e. $i_{\pi(n)} \in I_{\pi(n)}$, one has $\left(i_{1}, i_{2}, \ldots, i_{n}\right) \notin E$.

The following two propositions from Hammond and Sun (2006b) show that one can extend both the finite product probability space $\mathcal{P}^{n}$ and the infinite product probability space $\mathcal{P}^{\infty}$ by including all iteratively null sets, then forming the iterated completion.

Proposition 3 Given any $n \in \mathbb{N}$, let $\mathcal{E}_{n}$ denote the family of all iteratively null sets in the $n$-fold product $\left(I^{n}, \mathcal{I}^{n}, \lambda^{n}\right)$. Then there exists a complete and countably additive probability space

$$
\overline{\mathcal{P}}^{n}:=\left(I^{n}, \overline{\mathcal{I}}^{n}, \bar{\lambda}^{n}\right):=\left(I^{n}, \bar{\bigotimes}_{k=1}^{n} \mathcal{I}_{k}, \bar{\bigotimes}_{k=1}^{n} \lambda_{k}\right)
$$


that satisfies the Fubini property, with:

1. $\overline{\mathcal{I}}^{n}$ as the $\sigma$-algebra $\sigma\left(\mathcal{I}^{n} \cup \mathcal{E}_{n}\right)$, which is equal to the collection

$$
\mathcal{I}^{n} \triangle \mathcal{E}_{n}:=\left\{D \triangle E: D \in \mathcal{I}^{n}, E \in \mathcal{E}_{n}\right\}
$$

2. $\bar{\lambda}^{n}$ as the unique measure that satisfies $\bar{\lambda}^{n}(D \triangle E):=\lambda^{n}(D)$ whenever $D \in \mathcal{I}^{n}$ and $E \in \mathcal{E}_{n}$.

Proposition 4 There exists a countably additive probability space

$$
\overline{\mathcal{P}}^{\infty}:=\left(I^{\infty}, \overline{\mathcal{I}}^{\infty}, \bar{\lambda}^{\infty}\right):=\left(I^{\infty}, \bar{\bigotimes}_{k=1}^{\infty} \mathcal{I}_{k}, \bar{\bigotimes}_{k=1}^{\infty} \lambda_{k}\right)
$$

in which

1. $\overline{\mathcal{I}}^{\infty}$ is the $\sigma$-algebra generated by the union $\mathcal{G}:=\cup_{n=1}^{\infty} \mathcal{G}_{n}$ of the families $\mathcal{G}_{n}$ of cylinder sets taking the form $G_{n}=A \times \prod_{k=n+1}^{\infty} I_{k}$ for some $A \in \overline{\mathcal{I}}^{n}$;

2. $\bar{\lambda}^{\infty}$ is the unique countably additive extension to $\overline{\mathcal{I}}^{\infty}$ of the set function $\mu: \mathcal{G} \rightarrow$ $[0,1]$ defined so that $\mu\left(A \times \prod_{k=n+1}^{\infty} I_{k}\right):=\bar{\lambda}^{n}(A)$ for all $A \in \overline{\mathcal{I}}^{n}$.

Moreover, for any $\bar{D} \in \overline{\mathcal{I}}^{\infty}$, there exist $D \in \mathcal{I}^{\infty}$ and $E \in \overline{\mathcal{I}}^{\infty}$ such that $\bar{D}=D \triangle E$ and $\bar{\lambda}^{\infty}(E)=0$.

Unlike the finite product $\overline{\mathcal{P}}^{n}$, the infinite product measure space $\overline{\mathcal{P}}^{\infty}$ in Proposition 4 may not be complete in the usual sense. One can always complete it by the usual procedure [see, for example, Dudley (1989, pp. 78-79)]. We still use the same notation to denote this completion, which also retains the property stated in the last sentence of Proposition 4.

The completed probability space $\overline{\mathcal{P}}^{\infty}$ will be called the iterated completion of $\mathcal{P}^{\infty}$, as well as the iteratively complete product of the spaces $\mathcal{P}_{k}(k \in \mathbb{N})$. Let $i^{\infty}=$ $\left(i_{1}, i_{2}, \ldots, i_{n}, \ldots\right)$ denote a general element of $I^{\infty}$.

Next, let $\mathcal{C}$ be a countably generated sub- $\sigma$-algebra of $\mathcal{F}$ in $(\Omega, \mathcal{F}, P)$, and $g$ a process from $I \times \Omega$ to $X$ that is regular conditionally independent given $\mathcal{C}$. The following two lemmas state (part of) the results in Theorem 1 and Proposition 2 in Hammond and Sun (2016) respectively.

Lemma 1 The process $g$ satisfies the one-way Fubini property.

Lemma 2 Let $h$ be any measurable function mapping the product space $(I \times \Omega, \mathcal{I} \otimes$ $\mathcal{F}, \lambda \otimes P)$ to a Polish space $Y$. Then, for $\lambda$-almost all $i \in I$, the two random variables $g_{i}$ and $h_{i}$ are conditionally independent given $\mathcal{C}$.

\subsection{Proof of Theorem 1}

First, let

$$
\begin{aligned}
D^{\infty}:=\left\{i^{\infty}=\right. & \left(i_{k}\right)_{k=1}^{\infty} \in I^{\infty}: \\
& \left.\left(g_{i_{k}}\right)_{k=1}^{\infty} \text { is mutually conditionally independent given } \mathcal{C}\right\}
\end{aligned}
$$


denote the set of all infinite sequences $i^{\infty} \in I^{\infty}$ such that the associated sequence of random variables $g_{i_{k}}(k \in \mathbb{N})$ are mutually conditionally independent given $\mathcal{C}$.

Next, for any $n \in \mathbb{N}$, let

$$
\begin{aligned}
& D^{n}:=\left\{i^{n}=\left\{i_{k}\right\}_{k=1}^{n} \in I^{n}:\right. \\
&\left.\left\{g_{i_{k}}\right\}_{k=1}^{n} \text { is mutually conditionally independent given } \mathcal{C}\right\}
\end{aligned}
$$

denote the projection of the set $D^{\infty} \subset I^{\infty}$ onto the finite subproduct set $I^{n}$ of all sequences of length $n$. Since $g$ is essentially pairwise conditionally independent given $\mathcal{C}$, and also admits an essentially regular conditional distribution process $\mu$ given $\mathcal{C}$, Theorem 1 in Hammond and Sun (2006b) implies that $D^{n} \in \overline{\mathcal{I}}^{n}$ and $\bar{\lambda}^{n}\left(D^{n}\right)=1$ for any $n \in \mathbb{N}$.

For each $n \in \mathbb{N}$, let

$$
E^{n}:=\left\{\left(i^{\infty}, j^{\infty}\right) \in I^{\infty} \times I^{\infty}:\left(i_{1}, \ldots, i_{n}, j_{1}, \ldots, j_{n}\right) \in D^{2 n}\right\}
$$

It is easy to see that $\overline{\bar{\lambda} \infty \otimes \bar{\lambda}^{\infty}}\left(E^{n}\right)=\bar{\lambda}^{2 n}\left(D^{2 n}\right)=1$. Let $E=\cap_{n=1}^{\infty} E^{n}$. It is clear that

$$
\overline{\bar{\lambda} \infty \otimes \bar{\lambda}^{\infty}}(E)=1
$$

Also, for any $i^{\infty} \in I^{\infty}$, let $G\left(i^{\infty}\right):=\left\{g_{i_{k}}: k \in \mathbb{N}\right\}$ denote the associated countable set of random variables. Then for any $\left(i^{\infty}, j^{\infty}\right) \in E$, we know that the random variables in the set $G\left(i^{\infty}\right) \cup G\left(j^{\infty}\right)$ are mutually conditionally independent given $\mathcal{C}$. It follows from (14) that, for $\bar{\lambda}^{\infty}$-a.e. $i^{\infty} \in I^{\infty}$, the random variables in $G\left(i^{\infty}\right) \cup G\left(j^{\infty}\right)$ are mutually conditionally independent given $\mathcal{C}$ for $\bar{\lambda}^{\infty}$-a.e. $j^{\infty} \in I^{\infty}$.

Note that the infinite product $\sigma$-algebra $\mathcal{B}^{\infty}$ is generated by the family of all infinite cylinder sets which, for some $n \in \mathbb{N}$ and some collection $B_{1}, \ldots B_{n} \in \mathcal{B}$ of $n$ Borel sets, take the form $\prod_{i=1}^{n} B_{i} \times X^{\infty}$. To prove that $\bar{\mu}$ is an essentially regular conditional distribution process given $\mathcal{C}$, it is enough to consider the $\pi$-system consisting of these cylinder sets.

Fix any $i^{\infty}=\left(i_{k}\right)_{k=1}^{\infty} \in D^{\infty}$, where $D^{\infty}$ was defined in (12). For any $B_{1}, \ldots B_{n} \in$ $\mathcal{B}$, mutual conditional independence given $\mathcal{C}$ of all the random variables in the sequence $\left(g_{i_{k}}\right)_{k=1}^{\infty}$ implies that for $P$-a.e. $\omega \in \Omega$ one has

$$
\begin{aligned}
& P\left(\left(G\left(i^{\infty}\right)\right)^{-1}\left(B_{1} \times \cdots \times B_{n} \times X^{\infty}\right) \mid \mathcal{C}\right)(\omega) \\
& \quad=P\left(\left(g_{i_{1}}, \ldots, g_{i_{n}}\right)^{-1}\left(B_{1} \times \cdots \times B_{n}\right) \mid \mathcal{C}\right)(\omega) \\
& \quad=P\left(g_{i_{1}}^{-1}\left(B_{1}\right) \mid \mathcal{C}\right)(\omega) \cdots P\left(g_{i_{n}}^{-1}\left(B_{n}\right) \mid \mathcal{C}\right)(\omega) \\
& \quad=\mu_{i_{1} \omega}\left(B_{1}\right) \cdots \mu_{i_{n} \omega}\left(B_{n}\right)
\end{aligned}
$$

But definition (3) implies that

$$
\mu_{i_{1} \omega}\left(B_{1}\right) \cdots \mu_{i_{n} \omega}\left(B_{n}\right)=\bar{\mu}_{i \infty \omega}\left(B_{1} \times \cdots \times B_{n} \times X^{\infty}\right)
$$


So this proves that $I^{\infty} \times \Omega \ni\left(i^{\infty}, \omega\right) \mapsto \bar{\mu}_{i}{ }_{\omega}$ is an essentially regular conditional distribution process of $G$ given $\mathcal{C}$. Therefore, Lemma 1 implies that there exists a one-way Fubini extension $\left(I^{\infty} \times \Omega, \tilde{\mathcal{W}}, \tilde{Q}\right)$ of $\left(I^{\infty} \times \Omega, \overline{\mathcal{I}}^{\infty} \otimes \mathcal{F}, \bar{\lambda}^{\infty} \otimes P\right)$ such that $G$ is $\tilde{\mathcal{W}}$-measurable.

\subsection{Proofs of the results in Sect. 4}

Proof of Proposition 1 Take as given the real-valued functions $h$ and $f$ specified at the start of Sect. 4, as well as the regular conditional process $I \times \Omega \ni(i, \omega) \mapsto \mu_{i \omega} \in$ $\mathcal{M}(X)$ defined in Sect. 3. For any $i \in I$ and $\omega \in \Omega$, let

$$
\varphi(i, \omega):=\int_{X} h_{i}(x) d \mu_{i \omega} \text { and } \psi(i, \omega):=f(i, \omega)-\varphi(i, \omega)
$$

We first prove that the random variable

$$
\Omega \ni \omega \mapsto \int_{I} \varphi(i, \omega) d \lambda=\int_{I}\left[\int_{X} h_{i}(x) d \mu_{i \omega}\right] d \lambda
$$

belongs to $L^{2}(P)$. The property of essentially regular conditional distribution processes implies that

$$
\text { for } \lambda \text {-almost all } i \in I \text {, one has } \varphi(i, \omega)=\mathbb{E}[f(i) \mid \mathcal{C}](\omega) P \text {-a.s. }
$$

Thus, by the Fubini property and Jensen's inequality, one has

$$
\begin{aligned}
\int_{\Omega}\left[\int_{I} \varphi(i, \omega) d \lambda\right]^{2} d P & =\int_{\Omega}\left[\int_{I} \mathbb{E}[f(i) \mid \mathcal{C}](\omega) d \lambda\right]^{2} d P \\
\leq \int_{\Omega}\left[\int_{I}(\mathbb{E}[f(i) \mid \mathcal{C}](\omega))^{2} d \lambda\right] d P & =\int_{I}\left[\int_{\Omega}(\mathbb{E}[f(i) \mid \mathcal{C}](\omega))^{2} d P\right] d \lambda \\
\leq \int_{I}\left[\int_{\Omega} \mathbb{E}\left[f^{2}(i) \mid \mathcal{C}\right](\omega) d P\right] d \lambda & =\int_{I}\left[\int_{\Omega} f^{2}(i, \omega) d P\right] d \lambda
\end{aligned}
$$

Because of our assumption that $\int_{I}\left[\int_{\Omega} h_{i}^{2}\left(g_{i}(\omega)\right) d P\right] d \lambda=\int_{I}\left[\int_{\Omega} f_{i}^{2}(\omega) d P\right] d \lambda$ is finite, the last integral is finite. This proves that $\omega \mapsto \int_{I} \varphi(i, \omega) d \lambda$ is also a function that belongs to $L^{2}(P)$. Also $\varphi$ can be viewed as essentially a function from $(I, \mathcal{I}, \lambda)$ to $L^{2}(\mathcal{C}, P)$, the space of real-valued, $\mathcal{C}$-measurable and square integrable functions on $(\Omega, \mathcal{F}, P)$.

Since $\mathcal{C}$ is countably generated, we know that $L^{2}(\mathcal{C}, P)$ is separable, which implies that $\varphi$ is $\lambda$-essentially separably valued. ${ }^{9}$ It is easy to see that $\varphi$ is also weakly $\lambda$ measurable. ${ }^{10}$ Then Theorem 2 in p. 42 of Diestel and Uhl (1977) implies that the function $i \mapsto \varphi(i)$ is $\lambda$-measurable. Hence, there exists a sequence of simple functions $i \mapsto \varphi^{k}(i)$ with $\lim _{k \rightarrow \infty}\left\|\varphi^{k}-\varphi\right\|=0$ for $\lambda$-a.e. $i \in I$. From (17) note that $\int_{I}\|\varphi(i)\|^{2} d \lambda=\int_{I}\left[\int_{\Omega}(\mathbb{E}[f(i) \mid \mathcal{C}](\omega))^{2} d P\right] d \lambda$. Thus, Jensen's inequality implies

\footnotetext{
9 See p. 42 in Diestel and Uhl (1977) for formal definitions.
}

10 See p. 41 in Diestel and Uhl (1977) for formal definitions. 
that

$$
\int_{I}\|\varphi(i)\|^{2} d \lambda \leq \int_{I} \int_{\Omega} \mathbb{E}\left[f^{2}(i) \mid \mathcal{C}\right] d P d \lambda=\int_{I} \int_{\Omega} f(i)^{2} d P d \lambda<\infty
$$

But then the Cauchy-Schwarz inequality implies that

$$
\int_{I}\|\varphi(i)\| d \lambda \leq\left(\int_{I}\|\varphi(i)\|^{2} d \lambda\right)^{1 / 2}<\infty
$$

By Theorem 2 on p. 45 of Diestel and Uhl (1977), we know that $\varphi$ viewed as a function from $(I, \mathcal{I}, \lambda)$ to $L^{2}(P)$ is Bochner integrable. Next, the classical law of large numbers for Bochner integrable functions, as shown in Beck (1963) and Mourier (1953) (see also Dobric 1987 and Hoffmann-Jørgensen 1985), says that for $\lambda^{\infty}$-a.e. $i^{\infty} \in I^{\infty}$, one has

$$
\lim _{n \rightarrow \infty}\left\|\frac{1}{n} \sum_{k=1}^{n} \varphi\left(i_{k}\right)-\int_{I} \int_{X} h_{i}(x) d \mu_{i \omega} d \lambda\right\|=0
$$

The proof of Lemma 11 in Hammond and Sun (2008) shows that there exists $D^{*} \in \overline{\mathcal{I}}^{\infty}$ with $\bar{\lambda}^{\infty}\left(D^{*}\right)=1$ such that for any $i^{\infty} \in D^{*}$, the random variables $\left(\psi_{i_{k}}\right)_{k=1}^{\infty}$ defined by (15) are mutually orthogonal. This implies that for any $i^{\infty} \in D^{*}$, we have

$$
\left\|\frac{1}{n} \sum_{k=1}^{n} \psi\left(i_{k}\right)\right\|^{2}=\frac{1}{n^{2}} \sum_{k=1}^{n}\left\|\psi\left(i_{k}\right)\right\|^{2}
$$

Since $\int_{I}\|\psi(i)\|^{2} d \lambda<\infty$, the usual strong law of large numbers implies that for $\lambda^{\infty}$-a.e. $i^{\infty} \in I^{\infty}$ one has

$$
\lim _{n \rightarrow \infty} \frac{1}{n} \sum_{k=1}^{n}\left\|\psi\left(i_{k}\right)\right\|^{2}=\int_{I}\|\psi(i)\|^{2} d \lambda
$$

It clearly follows that for $\bar{\lambda}^{\infty}$-a.e. $i^{\infty} \in I^{\infty}$, one has

$$
\lim _{n \rightarrow \infty}\left\|\frac{1}{n} \sum_{k=1}^{n} \psi\left(i_{k}\right)\right\|^{2}=0
$$

Combining Eqs. (18) and (21), while using definition (15) of the function $i \mapsto \psi(i) \in$ $\mathbb{R}$, it follows that for $\bar{\lambda}^{\infty}$-a.e. $i^{\infty} \in I^{\infty}$, one has 


$$
\begin{aligned}
& \lim _{n \rightarrow \infty}\left\|\frac{1}{n} \sum_{k=1}^{n} f\left(i_{k}\right)-\int_{I} \int_{X} h_{i}(x) d \mu_{i \omega}(x) d \lambda\right\| \\
& =\lim _{n \rightarrow \infty}\left\|\frac{1}{n} \sum_{k=1}^{n} \psi\left(i_{k}\right)+\frac{1}{n} \sum_{k=1}^{n} \varphi\left(i_{k}\right)-\int_{I} \int_{X} h_{i}(x) d \mu_{i \omega}(x) d \lambda\right\|=0
\end{aligned}
$$

This completes the proof.

The following lemma is a special case of a result in Hammond and Sun (2008), which generalizes part of Lemma 2.1 in Hoffmann-Jørgensen (1985, p. 304) to the setting of iteratively complete product spaces.

Lemma 3 For each $n \in \mathbb{N}$, let $S_{n}$ be a subset of I whose $\lambda$-outer measure is one. Then the $\bar{\lambda}^{\infty}$-outer measure of $\prod_{n=1}^{\infty} S_{n}$ is also one.

The next lemma is also taken from Hammond and Sun (2008). It generalizes to iteratively complete products one part of Theorem 2.4 in (Hoffmann-Jørgensen 1985, p. 310), which is due to Talagrand.

Lemma 4 Let $g$ be a real-valued function on $(I, \mathcal{I}, \lambda)$. Suppose there is a real constant c such that

$$
\lim _{n \rightarrow \infty} \frac{g\left(i_{1}\right)+\cdots+g\left(i_{n}\right)}{n}=c \text { for } \bar{\lambda}^{\infty} \text {-a.e. } i^{\infty} \in I^{\infty}
$$

Then $g$ is $\lambda$-integrable, with $\int_{I} g(i) d \lambda=c$.

The proof of the following lemma adapts some of the ideas used in the proofs of Lemma 2.1 and Theorem 2.4 in Hoffmann-Jørgensen (1985), and of Lemma 1.4 in Hoffmann-Jørgensen (1977).

Lemma 5 If a function $f$ from I to a Banach space $\mathbb{B}$ satisfies SLLN, then it is norm integrably bounded.

Proof Let $f \in \operatorname{SLLN}(\mathbb{B})$, with $\left\|a-\frac{1}{n} \sum_{k=1}^{n} f\left(i_{k}\right)\right\| \rightarrow 0$ for $\bar{\lambda}^{\infty}$-a.e. $i^{\infty} \in I^{\infty}$. Let $D$ be the set of all $i^{\infty} \in I^{\infty}$ such that $\left\|\frac{1}{n} f\left(i_{n}\right)\right\| \rightarrow 0$ as $n \rightarrow \infty$. Because of the decomposition

$$
\frac{1}{n} f\left(i_{n}\right)=-\left[a-\frac{1}{n} \sum_{k=1}^{n} f\left(i_{k}\right)\right]+\frac{n-1}{n}\left[a-\frac{1}{n-1} \sum_{k=1}^{n-1} f\left(i_{k}\right)\right]+\frac{1}{n} a
$$

it follows that

$$
\left\|\frac{1}{n} f\left(i_{n}\right)\right\| \leq\left\|a-\frac{1}{n} \sum_{k=1}^{n} f\left(i_{k}\right)\right\|+\frac{n-1}{n}\left\|a-\frac{1}{n-1} \sum_{k=1}^{n-1} f\left(i_{k}\right)\right\|+\frac{1}{n}\|a\|
$$

Now each term on the right-hand side of (23) converges $\bar{\lambda}^{\infty}$-a.s. to 0 , so $\bar{\lambda}^{\infty}(D)=1$.

Let $i \mapsto g(i)$ be an upper $\lambda$-envelope of $i \mapsto\|f(i)\|$, in the sense that $g: I \rightarrow$ $\mathbb{R}_{+} \cup\{\infty\}$ is an $\mathcal{I}$-measurable function satisfying: (i) $g(i) \geq\|f(i)\|$ for all $i \in I$; (ii) 
for any $\mathcal{I}$-measurable function $h$ from $I$ to $\mathbb{R}_{+} \cup\{\infty\}$, the $\lambda$-inner measure of the set $\{i \in I:\|f(i)\| \leq h(i)<g(i)\}$ is zero (see Hoffmann-Jørgensen 1985, p. 302). For each $n \in \mathbb{N}$, define

$$
S_{n}:=\{i \in I: g(i) \leq 2\|f(i)\| \text { or }\|f(i)\| \geq n\}
$$

Define the function $h_{n}:=\min \left\{n, \frac{1}{2} g\right\}$ on $I$, which is evidently $\mathcal{I}$-measurable. Also, it is clear that $\|f(i)\|<h_{n}(i)<g(i)$ for all $i \in I \backslash S_{n}$ (even when $g(i)=\infty$ ). By definition of the upper $\lambda$-envelope, therefore, the set $I \backslash S_{n}$ must have $\lambda$-inner measure zero, implying that its $\lambda$-outer measure of $S_{n}$ is one. Lemma 3 says that then the set $\prod_{n=1}^{\infty} S_{n}$ also has $\bar{\lambda}^{\infty}$-outer measure one, and so therefore does $D \cap \prod_{n=1}^{\infty} S_{n}$.

Fix any $i^{\infty} \in D \cap \prod_{n=1}^{\infty} S_{n}$. Since $\left\|\frac{1}{n} f\left(i_{n}\right)\right\| \rightarrow 0$ as $n \rightarrow \infty$, one must have $\left\|f\left(i_{n}\right)\right\|<n$ for sufficiently large $n$, and then $i_{n} \in S_{n}$ implies that $0 \leq g\left(i_{n}\right) \leq$ $2\left\|f\left(i_{n}\right)\right\|$. Hence, $\frac{1}{n} g\left(i_{n}\right) \rightarrow 0$. But $g$ is $\mathcal{I}$-measurable by definition, so $\frac{1}{n} g\left(i_{n}\right) \rightarrow 0$ for all $i^{\infty}$ in some $\mathcal{I}^{\infty}$-measurable superset $E$ of $D \cap \prod_{n=1}^{\infty} S_{n}$. Since the $\bar{\lambda}^{\infty}$-outer measure of $D \cap \prod_{n=1}^{\infty} S_{n}$ is one, it follows that $\bar{\lambda}^{\infty}(E)=\lambda^{\infty}(E)=1$.

Given any $i^{\infty} \in I^{\infty}$, let $\phi\left(i^{\infty}\right):=\sup _{n \in \mathbb{N}} \frac{1}{n} g\left(i_{n}\right)$. Then $\phi\left(i^{\infty}\right)$ is finite for all $i^{\infty} \in E$. Because $g$ is $\mathcal{I}$-measurable, the function $\phi: I \rightarrow \mathbb{R}_{+} \cup\{\infty\}$ must be $\mathcal{I}^{\infty}$-measurable. So there exists a positive integer $K$ such that

$$
\lambda^{\infty}\left(\left\{i^{\infty} \in I^{\infty}: \phi\left(i^{\infty}\right)<K\right\}\right)>\frac{1}{2}
$$

For each $n \in \mathbb{N}$, let $\alpha_{n}:=\lambda(\{i \in I: g(i) \geq n K\})$. Because $\lambda^{\infty}$ is a product measure, it is evident that

$$
\lambda^{\infty}\left(\left\{i^{\infty} \in I^{\infty}: \phi\left(i^{\infty}\right)<K\right\}\right)=\prod_{n=1}^{\infty}\left(1-\alpha_{n}\right)
$$

Obviously (24) and (25) imply that $\prod_{n=1}^{\infty}\left(1-\alpha_{n}\right)>\frac{1}{2}$. But $\ln \left(1-\alpha_{n}\right) \leq-\alpha_{n}$, so

$$
\sum_{n=1}^{\infty} \alpha_{n} \leq-\sum_{n=1}^{\infty} \ln \left(1-\alpha_{n}\right)<-\ln (1 / 2)=\ln 2<\infty
$$

This implies that $\lim _{n \rightarrow \infty} \alpha_{n}=0$, and so $\lambda(\{i \in I: g(i)=\infty\})=0$.

Given any fixed $i \in I$ with $g(i)<\infty$, let $m$ be the smallest integer such that $g(i)<$ $m K$. Then $g(i) \in[n K, \infty)$ for $n \in\{1, \ldots, m-1\}$, and so $\sum_{n=1}^{\infty} 1_{[n K, \infty)}(g(i))=$ $m-1$. It follows that

$$
g(i) \leq K+K \sum_{n=1}^{\infty} 1_{[n K, \infty)}(g(i))
$$

for all $i \in I$ with $g(i)<\infty$. Because $\lambda(\{i \in I: g(i)=\infty\})=0$, the definition of $\alpha_{n}$ implies that $\int_{I} 1_{[n K, \infty)}(g(i)) d \lambda=\alpha_{n}$. It follows from (26) and (27), therefore, that

$$
\int_{I} g d \lambda \leq K+K \sum_{n=1}^{\infty} \alpha_{n}<K(1+\ln 2)<\infty
$$


Finally, let $f^{*}$ be the function from $I$ to $\mathbb{R}_{+}$such that $f^{*}(i)=g(i)$ when $g(i)<\infty$ and $f^{*}(i)=0$ when $g(i)=\infty$. Clearly $f^{*}$ is a norm dominant $\lambda$-integrable function for $\|f\|$, so $f$ is norm integrably bounded.

Two functions $f$ and $\tilde{f}$ from $(I, \mathcal{I}, \lambda)$ to a Banach space $\mathbb{B}$ are said to be scalarly equivalent if, for any $b^{\prime} \in \mathbb{B}^{\prime}$, the corresponding real-valued functions $i \mapsto\left\langle f(i), b^{\prime}\right\rangle$ and $i \mapsto\left\langle\tilde{f}(i), b^{\prime}\right\rangle$ are equal for $\lambda$-a.e. $i \in I$.

Lemma 6 Let $\mathbb{H}$ be a Hilbert space and $f$ a function in $\mathcal{L}(\lambda, \mathbb{H})$ that is scalarly equivalent to the zero function. Then

$$
\lim _{n \rightarrow \infty}\left\|\frac{1}{n} \sum_{k=1}^{n} f\left(i_{k}\right)\right\|=0 \text { for } \bar{\lambda}^{\infty} \text {-a.e. } i^{\infty} \in I^{\infty}
$$

Proof Given $f \in \mathcal{L}(\lambda, \mathbb{H})$, let $g: I \rightarrow \mathbb{R}_{+}$be a $\lambda$-integrable function that norm dominates $f$. For each $k \in \mathbb{N}$, let $X_{k}$ be the random variable defined on $\left(I^{\infty}, \mathcal{I}^{\infty}, \lambda^{\infty}\right)$ by $X_{k}\left(i^{\infty}\right):=\left[g\left(i_{k}\right)\right]^{2}$. Since $\mathbb{E} X_{1}^{1 / 2}<\infty$ and the variables $X_{k}$ are i.i.d., the Marcinkiewicz-Zygmund Theorem for the case $p=1 / 2$ and $c=0$ (Chow and Teicher 1997, p. 125) implies that $n^{-2} \sum_{k=1}^{n} X_{k}\left(i^{\infty}\right) \rightarrow 0$ for $\lambda^{\infty}$-a.e. $i^{\infty} \in I^{\infty}$. Because the definition of $g$ implies that $\|f(i)\| \leq g(i)$ for all $i \in I$, we have $\left\|f\left(i_{k}\right)\right\|^{2} \leq\left[g\left(i_{k}\right)\right]^{2}=X_{k}\left(i^{\infty}\right)$ for all $k \in \mathbb{N}$. It follows that $n^{-2} \sum_{k=1}^{n}\left\|f\left(i_{k}\right)\right\|^{2} \rightarrow 0$ for $\lambda^{\infty}$-a.e. $i^{\infty} \in I^{\infty}$.

Next, we follow the idea behind some of the computations in the proof of Theorem 1.3 in Dobric (1987, p. 277). For any $i^{\infty} \in I^{\infty}$, we have

$$
\left\|\frac{1}{n} \sum_{k=1}^{n} f\left(i_{k}\right)\right\|^{2}=\frac{1}{n^{2}} \sum_{k=1}^{n}\left\|f\left(i_{k}\right)\right\|^{2}+\frac{2}{n^{2}} \sum_{1 \leq j<k \leq n}\left\langle f\left(i_{j}\right), f\left(i_{k}\right)\right\rangle
$$

Because $f$ is scalarly equivalent to zero, for any $h \in \mathbb{H}$ one has $\langle f(i), h\rangle=0$ for $\lambda$-a.e. $i \in I$. In particular, for any $i^{\prime} \in I$, one has $\left\langle f(i), f\left(i^{\prime}\right)\right\rangle=0$ for $\lambda$-a.e. $i \in I$. Hence there exists a $\overline{\mathcal{I}}^{2}$-measurable set $D \subseteq I \times I$ such that $\bar{\lambda}^{2}(D)=1$ and $\left\langle f(i), f\left(i^{\prime}\right)\right\rangle=0$ for all $\left(i, i^{\prime}\right) \in D$. For each pair $j, k \in \mathbb{N}$, let $D_{j k}$ denote the set of all sequences $i^{\infty} \in I^{\infty}$ such that $\left(i_{j}, i_{k}\right) \in D$, and define $D^{*}:=\cap_{j=1}^{\infty} \cap_{k=j+1}^{\infty} D_{j k}$. Then for all $i^{\infty} \in D^{*}$ one has $\left\langle f\left(i_{j}\right), f\left(i_{k}\right)\right\rangle=0$ for all $j, k \in \mathbb{N}$ with $j<k$. Obviously $D_{j k} \in \overline{\mathcal{I}}^{\infty}$ and $\bar{\lambda}^{\infty}\left(D_{j k}\right)=1$ for each $j, k \in \mathbb{N}$, so $D^{*} \in \overline{\mathcal{I}}^{\infty}$ and $\bar{\lambda}^{\infty}\left(D^{*}\right)=1$ also.

Combining the results in the last two paragraphs shows that (29) implies (28).

Proof of Theorem 2 Let $h$ be a function from $I$ to the Banach space $\mathbb{B}$ such that, for some $a \in \mathbb{B}$, one has $\lim _{n \rightarrow \infty}\left\|a-\frac{1}{n} \sum_{k=1}^{n} h\left(i_{k}\right)\right\|=0$ for $\bar{\lambda}^{\infty}$-a.e. $i^{\infty} \in I^{\infty}$. Take any fixed $b^{\prime} \in \mathbb{B}^{\prime}$, and let $I \ni i \mapsto g(i) \in \mathbb{R}$ be defined so that $g(i):=\left\langle h(i), b^{\prime}\right\rangle$ for all $i \in I$. A routine calculation shows that, for $\bar{\lambda}^{\infty}$-a.e. $i^{\infty} \in I^{\infty}$, one has

$$
\lim _{n \rightarrow \infty} \frac{1}{n}\left[g\left(i_{1}\right)+\cdots+g\left(i_{n}\right)\right]=\left\langle a, b^{\prime}\right\rangle
$$


Then Lemma 4 implies that $g$ is $\lambda$-integrable, with $\int_{I} g(i) d \lambda=\left\langle a, b^{\prime}\right\rangle$. Hence, $h$ is Gel'fand integrable and has $a$ as its Gel'fand integral. Lemma 5 implies that $h$ is also norm integrably bounded.

Now suppose that $\mathbb{B}$ is a Hilbert space $\mathbb{H}$. Let $f$ be any function in $\mathcal{L}(\lambda, \mathbb{H})$. Since $f$ is Gel'fand integrable, it follows from Aliprantis and Border (1999, Theorem 11.51) or Diestel and Uhl (1977, p. 52) that for each $S \in \mathcal{I}$, the function $i \mapsto\left(1_{S} f\right)(i)=$ $1_{S}(i) f(i)$ is Gel'fand integrable, where $i \mapsto 1_{S}(i) \in\{0,1\}$ is the indicator function of the measurable set $S$. Let $v(S)$ denote its Gel'fand integral over $I$, which is an element of $\mathbb{H}$. It follows that $\|v(S)\|^{2}=\langle v(S), v(S)\rangle=\int_{I}\left\langle\left(1_{S} f\right)(i), v(S)\right\rangle d \lambda$. By the hypothesis of norm integrable boundedness, there exists a $\lambda$-integrable function $f^{*}: I \rightarrow \mathbb{R}_{+}$such that $\|f(i)\| \leq f^{*}(i)$ for $\lambda$-a.e. $i \in I$, and so $\left\langle\left(1_{S} f\right)(i), v(S)\right\rangle \leq$ $\left(1_{S} f^{*}\right)(i)\|v(S)\|$. Hence $\|v(S)\|^{2} \leq \int_{I}\left(1_{S} f^{*}\right)(i)\|v(S)\| d \lambda$. So even when $v(S)=0$, one has

$$
\|v(S)\| \leq \int_{S} f^{*}(i) d \lambda
$$

Let $S_{1}, S_{2}, \ldots \in \mathcal{T}$ be any countable collection of pairwise disjoint measurable subsets of $T$. Obviously $v\left(\cup_{k=1}^{n} S_{k}\right)=\sum_{k=1}^{n} v\left(S_{k}\right)$ for $n=1,2, \ldots$. Furthermore, (30) implies that

$$
\sum_{k=1}^{n}\left\|v\left(S_{k}\right)\right\| \leq \sum_{k=1}^{n} \int_{S_{k}} f^{*}(i) d \lambda \leq \int_{T} f^{*}(i) d \lambda<+\infty
$$

It follows that the sequence defined by $s_{n}:=v\left(\cup_{k=1}^{n} S_{k}\right)=\sum_{k=1}^{n} v\left(S_{k}\right)$ is a Cauchy sequence, and so convergent in the complete normed space $\mathbb{H}$. Hence $v\left(\cup_{k=1}^{\infty} S_{k}\right)=$ $\sum_{k=1}^{\infty} v\left(S_{k}\right)$. It follows from (31) that $v$ is an $\mathbb{H}$-valued $\sigma$-additive measure of bounded variation. Moreover, (30) also implies that the vector measure $v$ is absolutely continuous w.r.t. $\lambda$.

Next, we shall show that $f$ is scalarly equivalent to a Bochner integrable function $\phi$ from $(I, \mathcal{I}, \lambda)$ to $\mathbb{H}$. Because the Hilbert space $\mathbb{H}$ is a particular kind of reflexive Banach space, it has the Radon-Nikodym property (Diestel and Uhl 1977, p. 82). So there exists a Bochner integrable function $\phi$ from $(I, \mathcal{I}, \lambda)$ to $\mathbb{H}$ such that $v(S)$ equals the Bochner integral $\int_{S} \phi(i) d \lambda$ for each $S \in \mathcal{I}$. Now the Bochner integral, when it exists, must equal the Gel'fand integral (Aliprantis and Border 1999, p. 423). So given any $h \in \mathbb{H}$, it follows that

$$
\langle v(S), h\rangle=\int_{S}\langle\phi(i), h\rangle d \lambda=\int_{S}\langle f(i), h\rangle d \lambda
$$

Because the choice of $S \in \mathcal{I}$ was arbitrary, one has $\langle f(i), h\rangle=\langle\phi(i), h\rangle$ for $\lambda$-a.e. $i \in I$. That is, $f$ is scalarly equivalent to $\phi .{ }^{11}$

Define $\psi:=f-\phi$. Because $\phi$ is Bochner integrable, it follows from Diestel and Uhl (1977, p. 45), for example, that $\|\phi\|$ is integrable. Clearly, then, $\psi$ is norm integrably

11 The argument used in this paragraph is essentially the same as the simple argument on (Diestel and Uhl 1977, p. 89), where the case of norm bounded functions is considered. See also Khan and Sun (1999) for discussion and for many additional references concerning this scalar equivalence result. 
bounded, Gel'fand integrable, and scalarly equivalent to zero. So Lemma 6 implies that $\psi \in \operatorname{LLN}(\mathbb{H})$. Then the classical law of large numbers for Bochner integrable functions, as shown in Beck (1963) and Mourier (1953) (see also Dobric (1987) and Hoffmann-Jørgensen (1985)), says that $\phi$ is in LLN(H), and so in $\operatorname{SLLN}(\mathbb{H})$ as well. Therefore $f=\phi+\psi \in \operatorname{SLLN}(\mathbb{H})$.

Proof of Claim 1 Let $\varphi$ be any square integrable random variable on $(\Omega, \mathcal{F}, P)$. By the property of regular conditional distribution process $\mu$ and the Fubini property, one has

$$
\int_{\Omega}\left[\int_{I} \int_{X} h(i, x) d \mu_{i \omega} d \lambda\right] \varphi(\omega) d P=\int_{I} \int_{\Omega} \mathbb{E}[f(i, \omega) \mid \mathcal{C}] \varphi(\omega) d P d \lambda
$$

Lemma 2 implies that for $\lambda$-almost all $i \in I$, the random variables $\omega \mapsto f_{i}(\omega)$ and $\omega \mapsto \varphi(\omega)$ are conditionally independent given $\mathcal{C}$. Therefore, we have

$$
\begin{aligned}
\int_{\Omega}\left[\int_{I} \int_{X} h(i, x) d \mu_{i \omega} d \lambda\right] \varphi(\omega) d P & =\int_{I} \int_{\Omega} \mathbb{E}[f(i, \omega) \varphi(\omega) \mid \mathcal{C}] d P d \lambda \\
& =\int_{I} \int_{\Omega} f(i, \omega) \varphi(\omega) d P d \lambda
\end{aligned}
$$

This implies that $f$ is Gel'fand integrable.

\subsection{Proof of Theorem 3}

By the usual existence result on Walrasian allocations in Aumann (1966) and Hildenbrand (1974), we know that there exists a Walrasian equilibrium $\left(x^{c}, p\right)$ for the economy $\mathcal{E}^{c}$. Because we assumed that the utility function $\mathbb{R}_{+}^{m} \ni x \mapsto u_{i}(x ; s)$ of each agent $i \in I$ is strictly monotonic, we know that for any $s \in S$, the Walrasian equilibrium price vector $p_{s}$ is strictly positive.

Note that, by assumption, the private signal process $I \times \Omega \ni(i, \omega) \mapsto f(i, \omega) \in T^{0}$ that was introduced in Sect. 5.1 is essentially pairwise conditionally independent given $\mathcal{C}$ and admits an essentially regular conditional distribution process $\mu$ given $\mathcal{C}$. Then Proposition 5 in Hammond and Sun (2008) implies that for $\bar{\lambda}^{\infty}$-a.e. $i^{\infty} \in I^{\infty}$, there exists $F \in \mathcal{F}$ with $P(F)=1$ such that for any $\omega \in F$ and any $q \in T_{0}$, one has

$$
\lim _{n \rightarrow \infty} \frac{1}{n} \sum_{k=1}^{n} 1_{q}\left(f\left(i_{k}, \omega\right)\right)=[\bar{\mu}(\omega)](q)
$$

The usual strong law of large numbers implies that for $\lambda^{\infty}$-a.e. $i^{\infty} \in I^{\infty}$ one has

$$
\begin{aligned}
\lim _{n \rightarrow \infty} \frac{1}{n} \sum_{k=1}^{n} e\left(i_{k}\right) & =\int_{I} e(i) d \lambda \\
\text { and } \quad \lim _{n \rightarrow \infty} \frac{1}{n} \sum_{k=1}^{n} x_{s}^{c}\left(i_{k}\right) & =\int_{I} x_{s}^{c}(i) d \lambda \text { for all } s \in S
\end{aligned}
$$


Let $D$ be the set of $i^{\infty} \in I^{\infty}$ such that the three Eqs. (32)-(34) all hold. It is clear that $\bar{\lambda}^{\infty}(D)=1$.

First, for any $i^{\infty} \notin D$ and $i \in I\left(i^{\infty}\right)$, construct $x^{i^{\infty}}(i, t):=e(i)$ for all $t \in T$. Also, for any $i^{\infty} \notin D$ and $i \in I\left(i^{\infty}\right)$, the definition of Walrasian equilibrium implies that the inequality $U_{i}^{i^{\infty}}\left(x_{i}^{i^{\infty}}, t_{i} \mid t_{i}\right) \geq U_{i}^{i^{\infty}}\left(x_{i}^{i^{\infty}}, t_{i}^{\prime} \mid t_{i}\right)$ holds for any $t_{i}, t_{i}^{\prime} \in T^{0}$.

Alternatively, consider any fixed $i^{\infty} \in D$. For any $n \in \mathbb{N}, t \in T$ and $q \in T_{0}$, let $\gamma_{n}^{i^{\infty}}(t, q):=\frac{1}{n} \sum_{k=1}^{\infty} 1_{\{q\}}\left(t_{i_{k}}\right)$. This defines a mapping $T \ni t \mapsto \gamma_{n}^{i^{\infty}}(t) \in \Delta\left(T^{0}\right)$. For any $t \in T$, given the counting measure $\bar{\gamma}$ on the finite set $T^{0}$, let

$$
\gamma^{i^{\infty}}(t):= \begin{cases}\lim _{n \rightarrow \infty} \gamma_{n}^{i^{\infty}}(t) & \text { if the limit exists } \\ \bar{\gamma} & \text { otherwise }\end{cases}
$$

Next, define the sets

$$
L_{s}^{i^{\infty}}:=\left\{t \in T: \gamma^{i^{\infty}}(t)=\mu_{s}\right\} \text { for all } s \in S \text {, and } L_{0}^{i^{\infty}}:=T \backslash \bigcup_{s \in S} L_{s}^{i^{\infty}}
$$

Because (32) holds $P$-a.s., it follows that $P_{s}^{T}\left(L_{s}^{i^{\infty}}\right)=1$.

Also, the non-triviality assumption implies that for any $s, s^{\prime} \in S$ with $s \neq s^{\prime}$, one has $L_{s}^{i^{\infty}} \cap L_{s^{\prime}}^{i^{\infty}}=\emptyset$. Thus, the collection $\left\{L_{0}^{i^{\infty}}\right\} \cup\left\{L_{s}^{i^{\infty}}: s \in S\right\}$ forms a measurable partition of $T$.

The definition (35) of $\gamma^{i^{\infty}}$ obviously implies that for any $i \in I\left(i^{\infty}\right)$ one has $\gamma^{i^{\infty}}\left(t_{-i}, t_{i}\right)=\gamma^{i^{\infty}}\left(t_{-i}, t_{i}^{\prime}\right)$ for all $t_{-i} \in T_{-i}$ and all $t_{i}, t_{i}^{\prime} \in T^{0}$. Hence, for any $i \in I\left(i^{\infty}\right), t \in T, t_{i}^{\prime} \in T^{0}$, and $s \in S$, one has

$$
t \in L_{s}^{i^{\infty}} \Longleftrightarrow \gamma^{i^{\infty}}(t)=\mu_{s} \Longleftrightarrow \gamma^{i^{\infty}}\left(t_{-i}, t_{i}^{\prime}\right)=\mu_{s} \Longleftrightarrow\left(t_{-i}, t_{i}^{\prime}\right) \in L_{s}^{i^{\infty}}
$$

Since $L_{0}^{i^{\infty}}$ equals $T \backslash \cup_{s \in S} L_{s}^{i^{\infty}}$, we also know that $t \in L_{0}^{i^{\infty}} \Longleftrightarrow\left(t_{-i}, t_{i}^{\prime}\right) \in L_{0}^{i^{\infty}}$. Hence, for any $i \in I\left(i^{\infty}\right)$ we have $x^{i^{\infty}}(i, t)=x^{i^{\infty}}\left(i,\left(t_{-i}, t_{i}^{\prime}\right)\right)$ for all $t \in T$ and $t_{i}^{\prime} \in T^{0}$. This trivially implies that for any $i \in I\left(i^{\infty}\right)$ and any $t_{i}, t_{i}^{\prime} \in T^{0}$, the allocation $I \times T \ni(i, t) \mapsto x^{i^{\infty}}(i, t) \in \mathbb{R}_{+}^{m}$ satisfies the corresponding incentive constraint

$$
U_{i}^{i^{\infty}}\left(x_{i}^{i^{\infty}}, t_{i} \mid t_{i}\right) \geq U_{i}^{i^{\infty}}\left(x_{i}^{i^{\infty}}, t_{i}^{\prime} \mid t_{i}\right)
$$

For each $s \in S$, let $\delta_{s}$ denote the Dirac measure on $S$ that gives probability one to the point $s$ and zero to all the other points of $S$. Define a function $H$ from $T$ to the space $\Delta(S)$ of all probability measures on the finite set $S$ by letting

$$
H(t):= \begin{cases}\delta_{s} & \text { for the unique } s \in S \text { such that } t \in L_{s}^{i \infty} \\ \delta_{s_{1}} & \text { for } t \in L_{0}^{i^{\infty}}\end{cases}
$$

Then the same proof as in Lemma 3 of Sun and Yannelis (2007) shows that for each $t \in T$, the measure $H(t)$ is a version of $P^{s}(\cdot \mid t)$.

Now we are ready to prove Claim 2. 
Proof of Claim 2 Fix any $i^{\infty} \in D$. Let $\mathcal{C}^{\prime}$ be the $\sigma$-algebra generated by the finite family $\left\{L_{s}^{i^{\infty}}: s \in S\right\}$. Note that for any $s \in S$, one has

$$
P\left(\tilde{t} \in L_{s}^{i^{\infty}}\right)=\sum_{s^{\prime} \in S} \pi_{s^{\prime}} P_{s^{\prime}}^{T}\left(L_{s}^{i^{\infty}}\right)=\pi_{s} P_{s}^{T}\left(L_{s}^{i^{\infty}}\right)=\pi_{s}
$$

and

$$
P\left(\tilde{s}=s \mid \tilde{t} \in L_{s}^{i^{\infty}}\right)=\frac{P\left(\tilde{s}=s, \tilde{t} \in L_{s}^{i^{\infty}}\right)}{P^{T}\left(L_{s}^{i^{\infty}}\right)}=\frac{\pi_{s} P\left(\tilde{t} \in L_{s}^{i^{\infty}} \mid \tilde{s}=s\right)}{\pi_{s} P_{s}^{T}\left(L_{s}^{i^{\infty}}\right)}=1
$$

Fix any $s \in S$, any $q, q^{\prime} \in T^{0}$, and any $i, j \in I$ such that the random variables $f_{i}$ and $f_{j}$ from $\Omega$ to $T^{0}$ are conditionally independent given $s$. We know that

$$
\begin{aligned}
P^{T}\left(g_{i}=q, g_{j}=q^{\prime} \mid L_{s}^{i^{\infty}}\right) & =\frac{P\left(f_{i}=q, f_{j}=q^{\prime}, \tilde{t} \in L_{s}^{i^{\infty}}\right)}{P^{T}\left(L_{s}^{i^{\infty}}\right)} \\
& =\frac{\pi_{s} P\left(f_{i}=q, f_{j}=q^{\prime}, \tilde{t} \in L_{s}^{i^{\infty}} \mid \tilde{s}=s\right)}{\pi_{s} P_{s}^{T}\left(L_{s}^{i^{\infty}}\right)}
\end{aligned}
$$

Because $P_{s}^{T}\left(L_{s}^{i^{\infty}}\right)=1$, whereas $f_{i}$ and $f_{j}$ are conditionally independent given $s$, we have

$$
\begin{aligned}
P^{T}\left(g_{i}=q, g_{j}=q^{\prime} \mid L_{s}^{i^{\infty}}\right) & =P\left(f_{i}=q, f_{j}=q^{\prime}, \tilde{t} \in L_{s}^{i^{\infty}} \mid \tilde{s}=s\right) \\
& =P\left(f_{i}=q, f_{j}=q^{\prime} \mid \tilde{s}=s\right) \\
& =P\left(f_{i}=q \mid \tilde{s}=s\right) \cdot P\left(f_{j}=q^{\prime} \mid \tilde{s}=s\right) \\
& =P^{T}\left(g_{i}=q \mid L_{s}^{i^{\infty}}\right) \cdot P^{T}\left(g_{j}=q^{\prime} \mid L_{s}^{i^{\infty}}\right)
\end{aligned}
$$

By Eqs. (39) and (40), we know that

$$
\begin{aligned}
& P\left(f_{i}=q \mid \tilde{s}=s\right) \cdot P\left(f_{j}=q^{\prime} \mid \tilde{s}=s\right) \\
& \quad=\frac{1}{\pi_{s}^{2}} P\left(f_{i}=q, \tilde{s}=s\right) \cdot P\left(f_{j}=q^{\prime}, \tilde{s}=s\right) \\
& \quad=\frac{1}{\pi_{s}^{2}} P\left(\tilde{t} \in L_{s}^{i^{\infty}}\right)^{2} \cdot P\left(f_{i}=q, \tilde{s}=s \mid \tilde{t} \in L_{s}^{i^{\infty}}\right) \cdot P\left(f_{j}=q^{\prime}, \tilde{s}=s \mid \tilde{t} \in L_{s}^{i^{\infty}}\right) \\
& \quad=P\left(f_{i}=q \mid \tilde{t} \in L_{s}^{i^{\infty}}\right) \cdot P\left(f_{j}=q^{\prime} \mid \tilde{t} \in L_{s}^{i^{\infty}}\right) \\
& \quad=P^{T}\left(g_{i}=q \mid L_{s}^{i^{\infty}}\right) \cdot P^{T}\left(g_{j}=q^{\prime} \mid L_{s}^{i^{\infty}}\right)
\end{aligned}
$$

This implies that $P^{T}\left(g_{i}=q, g_{j}=q^{\prime} \mid L_{s}^{i^{\infty}}\right)=P^{T}\left(g_{i}=q \mid L_{s}^{i^{\infty}}\right) \cdot P^{T}\left(g_{j}=q^{\prime} \mid L_{s}^{i^{\infty}}\right)$. Hence, $g$ is essentially pairwise conditionally independent given $\mathcal{C}^{\prime}$.

For any $i \in I, s \in S$ and $t \in L_{s}^{i^{\infty}}$, let $\nu_{i t}$ denote $\mu_{i(s, t)}$, where $\mu$ is the essentially regular conditional distribution process of $f$ given $\mathcal{C}$. It is clear that $v$ is an essentially regular conditional distribution process of $g$ given $\mathcal{C}^{\prime}$. Therefore, $g$ is regularly conditionally independent given $\mathcal{C}^{\prime}$. This completes the proof of Claim 2. 
We now continue the proof of Theorem 3 .

Let $E_{s}^{i^{\infty}}$ be the set of all $t \in L_{s}^{i^{\infty}}$ such that $P^{T}(\cdot \mid t)=\delta_{s}$. Clearly $P_{s}^{T}\left(E_{s}^{i^{\infty}}\right)=1$ for any $t \in E_{s}^{i^{\infty}}$. Let $E^{i^{\infty}}:=\cup_{s \in S} E_{s}^{i^{\infty}}$. Then

$$
P^{T}\left(E^{i^{\infty}}\right)=\sum_{s^{\prime} \in S} \pi_{s^{\prime}} P_{s^{\prime}}^{T}\left(\cup_{s \in S} E_{S}^{i^{\infty}}\right)=\sum_{s^{\prime} \in S} \pi_{s^{\prime}} P_{s^{\prime}}^{T}\left(E_{s^{\prime}}^{i^{\infty}}\right)=\sum_{s^{\prime} \in S} \pi_{s^{\prime}}=1
$$

Given the Walrasian equilibrium allocation $(i, s) \mapsto x_{s}^{c}(i)$ for the economy $\mathcal{E}^{c}$, as specified by (6), define a mapping $x^{i^{\infty}}$ from $I \times T$ to $\mathbb{R}_{+}^{m}$ by letting

$$
x^{i^{\infty}}(i, t):= \begin{cases}x_{s}^{c}(i) & \text { if there is a unique } s \in S \text { such that } t \in L_{s}^{i^{\infty}} \\ e(i) & \text { if } t \in L_{0}^{i^{\infty}}\end{cases}
$$

It is clear that $x^{i^{\infty}}$ only depends on reports from agents $i \in I\left(i^{\infty}\right)$. Hence $x^{i^{\infty}}$ is an allocation in the economy $\mathcal{E}^{i^{\infty}}$.

Note that for any $s \in S$ the feasibility condition in Part 2 of Definition 4 implies that $\int_{I} x_{s}^{c}(i) d \lambda=\int_{I} e(i) d \lambda$, and also

$$
\begin{aligned}
& \lim _{n \rightarrow \infty} \frac{1}{n} \sum_{k=1}^{n} x^{i^{\infty}}\left(i_{k}, t\right)=\lim _{n \rightarrow \infty} \frac{1}{n} \sum_{k=1}^{n} e\left(i_{k}\right)=\int_{I} e(i) d \lambda, \text { if } t \in L_{0}^{i^{\infty}} \\
& \lim _{n \rightarrow \infty} \frac{1}{n} \sum_{k=1}^{n} x^{i^{\infty}}\left(i_{k}, t\right)=\lim _{n \rightarrow \infty} \frac{1}{n} \sum_{k=1}^{n} x_{s}^{c}\left(i_{k}\right)=\int_{I} x_{s}^{c}(i) d \lambda, \text { if } t \in L_{s}^{i^{\infty}}
\end{aligned}
$$

These last equalities imply that, for any $t \in T$, as $n \rightarrow \infty$, the allocation defined by (41) satisfies the asymptotic feasibility condition

$$
\left\|\frac{1}{n} \sum_{k=1}^{n}\left[x^{i^{\infty}}\left(i_{k}, t\right)-e\left(i_{k}\right)\right]\right\| \rightarrow \int_{I}\left[x_{s}^{c}(i)-e(i)\right] d \lambda=0
$$

Now fix any $s \in S$ and $t \in E_{s}^{i^{\infty}}$. Evidently definition (7) implies that for any $i \in I$ one has $e(i) \in B_{i}\left(p_{s}\right)$. Since $P^{T}(\cdot \mid t)=\delta_{s}$, it follows from (8) that for any $i \in I\left(i^{\infty}\right)$, one has

$$
U_{i}^{i^{\infty}}\left(x^{i^{\infty}}(i, t) \mid t\right)=u_{i}\left(x_{s}^{c}(i) ; s\right) \geq u_{i}(e(i) ; s)=U_{i}^{i^{\infty}}(e(i) \mid t) .
$$

This proves ex post individual rationality.

Finally, fix any $\epsilon>0$. By uniform equicontinuity of the family of utility functions $\mathbb{R}_{+}^{m} \ni x \mapsto u_{i}(x ; s)$ (for $i \in I$ and $s \in S$ ), there exists $\delta>0$ such that whenever $x, x^{\prime} \in \mathbb{R}_{+}^{m}$ satisfy $\left\|x-x^{\prime}\right\|<\delta$, then $\left|u_{i}(x ; s)-u_{i}\left(x^{\prime} ; s\right)\right|<\epsilon$ for all $i \in I$ and $s \in S$.

Let $\bar{p}_{s}:=\min _{j \in\{1,2, \ldots, m\}} p_{s j}$ and $\delta^{\prime}:=\frac{1}{2} \bar{p}_{s} \delta$. For any $i \in I$ and $s \in S$, let $M_{s}^{i}:=p_{s} \cdot e(i)$ denote the value of agent $i$ 's endowment at the equilibrium price vector $p_{s}$ that applies in the economy $\mathcal{E}_{s}^{c}$. 
Fix any $i \in I\left(i^{\infty}\right)$ and $x \in B\left(p_{s}, M_{s}^{i}+\delta^{\prime}\right)$. Let $x^{\prime}=\frac{M_{s}^{i}}{M_{s}^{i}+\delta^{\prime}} x$. It is clear that $x^{\prime} \in B\left(p_{s}, M_{s}^{i}\right)$. By the definition of $\bar{p}_{s}$ and $\delta^{\prime}$, we have

$$
\begin{aligned}
& \left\|x-x^{\prime}\right\|=\frac{\delta^{\prime}}{M_{s}^{i}}\left\|x^{\prime}\right\| \leq \frac{\delta^{\prime}}{p_{s} \cdot x^{\prime}(i)}\left\|x^{\prime}\right\| \\
& \leq \frac{\delta^{\prime}}{\bar{p}_{s} \cdot \sum_{j=1}^{m} x_{j}^{\prime}(i)}\left\|x^{\prime}\right\| \leq \frac{\delta^{\prime}}{\bar{p}_{s} \cdot \sum_{j=1}^{m} x_{j}^{\prime}(i)} \sum_{j=1}^{m} x_{j}^{\prime}(i)=\frac{1}{2} \delta<\delta
\end{aligned}
$$

This implies that $u_{i}(x ; s)<u_{i}\left(x^{\prime} ; s\right)+\epsilon$. For any $i \in I\left(i^{\infty}\right)$ and any $x \in B\left(p_{s}, M_{s}^{i}+\right.$ $\left.\delta^{\prime}\right)$, it follows that

$$
U_{i}^{i^{\infty}}(x \mid t)<U_{i}^{i^{\infty}}\left(x_{i}^{i^{\infty}} \mid t\right)+\epsilon
$$

Let $I\left(i^{\infty}\right) \ni i \mapsto y_{i} \rightarrow \mathbb{R}_{+}^{m}$ be any sequence such that $U_{i}^{i^{\infty}}\left(y_{i} \mid t\right) \geq U_{i}^{i^{\infty}}\left(x_{i}^{i^{\infty}} \mid t\right)+\epsilon$ for all $i \in I\left(i^{\infty}\right)$. From (44) it follows that $p_{s} \cdot y_{i} \geq M_{s}^{i}+\delta^{\prime}$ for all $i \in I\left(i^{\infty}\right)$, which implies that $\frac{1}{n} \sum_{k=1}^{n} p_{s} \cdot\left[y_{i_{k}}-e\left(i_{k}\right)\right]$ does not converge to 0 . It is clear then that no sequence $I\left(i^{\infty}\right) \ni i \mapsto y_{i} \rightarrow \mathbb{R}_{+}^{m}$ with the property that $U_{i}^{i^{\infty}}\left(y_{i} \mid t\right) \geq U_{i}^{i^{\infty}}\left(x_{i}^{i^{\infty}} \mid t\right)+\epsilon$ for all $i \in I\left(i^{\infty}\right)$ can satisfy the asymptotic feasibility condition (42).

Finally, note that $G: I^{\infty} \times T \rightarrow\left(T^{0}\right)^{\infty}$ is the Monte Carlo sampling process based on $g$, and that $\left(I^{\infty} \times T, \tilde{\mathcal{W}}, \tilde{Q}\right)$ is a one-way Fubini extension of $\left(I^{\infty} \times T, \overline{\mathcal{I}}^{\infty} \otimes \mathcal{T}, \bar{\lambda}^{\infty} \otimes P^{T}\right)$ such that $G$ is $\tilde{\mathcal{W}}$-measurable. Within the framework of this one-way Fubini extension, the arguments in this section establish that the allocation mechanism $\left(i^{\infty}, i, t\right) \mapsto x^{i^{\infty}}(i, t)$ is incentive compatible, asymptotically feasible, ex post individually rational, and asymptotically Pareto efficient.

\section{References}

Aliprantis, C.D., Border, K.C.: Infinite Dimensional Analysis: A Hitchhiker's Guide, 2nd edn. Springer, New York (1999)

Aumann, R.J.: Markets with a continuum of traders. Econometrica 32, 39-50 (1964)

Aumann, R.J.: Existence of competitive equilibria in markets with a continuum of traders. Econometrica 34, 1-17 (1966)

Beck, A.: On the strong law of large numbers. In: Wright, F.B. (ed.) Ergodic Theory. Academic Press, New York (1963)

Bewley, T.F.: Stationary monetary equilibrium with a continuum of independently fluctuating consumers. In: Hildenbrand, W., Mas-Colell, A. (eds.) Contributions to Mathematical Economics: In Honor of Gérard Debreu, pp. 79-103. Elsevier, Amsterdam (1986) (Chap. 5)

Chow, Y.S., Teicher, H.: Probability Theory: Independence, Interchangeability, Martingales, 3rd edn. Springer, New York (1997)

Dasgupta, P., Hammond, P., Maskin, E.: Implementation of social choice rules: some general results on incentive compatibility. Rev. Econ. Stud. 46, 185-216 (1979)

Diestel, J., Uhl, J.J.: Vector Measures. American Mathematical Society, Rhode Island (1977)

Dobric, V.: The law of large numbers, examples and counterexamples. Math. Scand. 60, 273-291 (1987)

Dudley, R.M.: Real Analysis and Probability. Chapman \& Hall, New York (1989)

Glycopantis, D., Yannelis, N.C.: Differential Information Economies. Springer, Berlin (2005)

Hammond, P.J.: Straightforward individual incentive compatibility in large economies. Rev. Econ. Stud. 46, 263-282 (1979) 
Hammond, P.J., Sun, Y.N.: Monte Carlo simulation of macroeconomic risk with a continuum of agents: the symmetric case. Econ. Theory 21, 743-766 (2003). https://doi.org/10.1007/978-3-662-05858-9_34

Hammond, P.J., Sun, Y.N.: Joint measurability and the one-way Fubini property for a continuum of independent random variables. Proc. Am. Math. Soc. 134, 737-747 (2006a)

Hammond, P.J., Sun, Y.N.: The essential equivalence of pairwise and mutual conditional independence. Probab. Theory Relat. Fields 135, 415-427 (2006b)

Hammond, P.J., Sun, Y.N.: Monte Carlo simulation of macroeconomic risk with a continuum of agents: the general case. Econ. Theory 36, 303-325 (2008). https://doi.org/10.1007/s00199-007-0279-7

Hammond, P.J., Sun, Y.N.: The one-way Fubini property and conditional independence: an equivalence result. Online Discussion Paper 22, Centre for Research in Economic Theory and its Applications (CRETA), University of Warwick (2016). Forthcoming in Advances In Mathematics

He, W., Yannelis, N.C.: Existence of Walrasian equilibria with discontinuous, non-ordered, interdependent and price-dependent preferences. Econ. Theory 61, 497-513 (2016). https://doi.org/10.1007/s00199015-0875-X

Hildenbrand, W.: Core and Equilibria of a Large Economy. Princeton University Press, Princeton (1974)

Hoffmann-Jørgensen, J.: Probability in Banach Space, Ecole d'Eté de Probabilités de Saint-Flour, VI-1976. Lecture Notes in Math., vol. 598, pp. 1-186. Springer, Berlin (1977)

Hoffmann-Jørgensen, J.: The law of large numbers for non-measurable and non-separable random elements. Astérisque 131, 299-356 (1985)

Khan, M.A., Sun, Y.N.: Weak measurability and characterizations of risk. Econ. Theory 13, 541-560 (1999). https://doi.org/10.1007/s001990050269

Lucas Jr., R.E., Prescott, E.C.: Equilibrium search and unemployment. J. Econ. Theory 7, 188-209 (1974). https://doi.org/10.1016/B978-0-12-214850-7.50037-1

McLean, R., Postlewaite, A.: Informational size and incentive compatibility. Econometrica 70, 2421-2453 (2002)

Mourier, E.: Eléments aléatoires dans un espace de Banach. Ann. Inst. H. Poincaré 13, 161-244 (1953)

Myerson, R.: Optimal coordination mechanisms in generalized principal-agent problems. J. Math. Econ. 10, 67-81 (1982)

Podczeck, K.: On existence of rich Fubini extensions. Econ. Theory 45, 1-22 (2010). https://doi.org/10. 1007/s00199-009-0458-9

Sun, Y.N.: The exact law of large numbers via Fubini extension and characterization of insurable risks. J. Econ. Theory 126, 31-69 (2006). https://doi.org/10.1016/j.jet.2004.10.005

Sun, Y.N., Yannelis, N.C.: Perfect competition in asymmetric information economies: compatibility of efficiency and incentives. J. Econ. Theory 134, 175-194 (2007). https://doi.org/10.1016/j.jet.2006.03. 001

Sun, Y.N., Yannelis, N.C.: Ex ante efficiency implies incentive compatibility. Econ. Theory 36, 35-55 (2008). https://doi.org/10.1007/s00199-007-0261-4

Sun, Y.N., Zhang, Y.C.: Individual risk and Lebesgue extension without aggregate uncertainty. J. Econ. Theory 144, 432-443 (2009). https://doi.org/10.1016/j.jet.2008.05.001

Publisher's Note Springer Nature remains neutral with regard to jurisdictional claims in published maps and institutional affiliations. 